Check for updates

Cite this: RSC Adv., 2021, 11, 35765

Received 9th June 2021

Accepted 21st October 2021

DOI: $10.1039 / d 1 r a 04461 c$

rsc.li/rsc-advances

\section{Newly synthesised oxime and lactone derivatives from Dipterocarpus alatus dipterocarpol as anti- diabetic inhibitors: experimental bioassay-based evidence and theoretical computation-based prediction $\dagger$}

\author{
Tran Thi Phuong Thao, tab $^{\mathrm{ab}}$ Than Q. Bui, (iD $\ddagger^{\mathrm{c}}$ Nguyen Thi Thanh Hai, ${ }^{\mathrm{c}}$ \\ Lam K. Huynh, (I) dh Phan Tu Quy, ${ }^{e}$ Nguyen Chi Bao, ${ }^{f}$ Nguyen Thi Dung, ${ }^{a}$ \\ Nguyen Linh Chi, ${ }^{\text {ab }}$ Tran Van Loc, ${ }^{\text {ab }}$ Irina E. Smirnova, ${ }^{9}$ Anastasiya V. Petrova, ${ }^{9}$ \\ Pham Thi Ninh, ${ }^{a b}$ Tran Van Sung ${ }^{\star a b}$ and Nguyen Thi Ai Nhung (iD) *c
}

\begin{abstract}
Dipterocarpus alatus-derived products are expected to exhibit anti-diabetes properties. Natural dipterocarpol (1) was isolated from Dipterocarpus alatus collected in Quang Nam province, Vietnam; afterwards, 20 derivatives including 13 oxime esters $(2$ and $3 a-3 m)$ and 7 lactones $(4,5,6 a-6 e)$ were semi-synthesised. Their inhibitory effects towards diabetes-related proteins were investigated experimentally ( $\alpha$-glucosidase) and computationally (3W37, 3AJ7, and PTP1B). Except for compound 2, the other 19 compounds $(3 a-3 m, 4,5$, and $6 a-6 d)$ are reported for the first time, which were modified at positions C-3, C-24 and C-25 of the dipterocarpol via imidation, esterification, oxidative cleavage and lactonisation reactions. A framework based on docking-QSARIS combination was proposed to predict the inhibitory behaviour of the ligand-protein complexes. Enzyme assays revealed the most effective $\alpha$ glucosidase inhibitors, which follow the order $5\left(\mathrm{IC}_{50}\right.$ of $\left.2.73 \pm 0.05 \mu \mathrm{M}\right)>6 \mathrm{c}\left(\mathrm{IC}_{50}\right.$ of $\left.4.62 \pm 0.12 \mu \mathrm{M}\right)>$ $6 \mathrm{e}\left(\mathrm{IC}_{50}\right.$ of $\left.7.31 \pm 0.11 \mu \mathrm{M}\right)$, and the computation-based analysis confirmed this, i.e., 5 (mass: 416.2 amu; polarisability: $52.4 \AA^{3}$; DS: $-14.9 \mathrm{kcal} \mathrm{mol}^{-1}$ ) > 6c (mass: $490.1 \mathrm{amu}$; polarisability: $48.8 \AA^{3}$; DS: $-13.7 \mathrm{kcal} \mathrm{mol}^{-1}$ ) > 6e (mass: $549.2 \mathrm{amu}$; polarisability: $51.6 \AA^{3}$; DS: $-15.2 \mathrm{kcal} \mathrm{mol}^{-1}$ ). Further theoretical justifications predicted 5 and $6 c$ as versatile anti-diabetic inhibitors. The experimental results encourage next stages for the development of anti-diabetic drugs and the computational strategy invites more relevant work for validation.
\end{abstract}

\section{Introduction}

Diabetes mellitus (DM) is increasing in humans worldwide, especially in middle-income countries. According to The World Health Organization (WHO), diabetes was directly responsible for about 1.5 million deaths in 2019 and it is predicted that the increasing death toll will cause this disorder to be the seventh leading cause of death by $2030 .^{1}$ It has also been evidenced that cardiovascular disease, blindness, kidney failure, stroke, and limb amputation are related to this disorder. ${ }^{2}$ In particular, the noninsulin-dependent disorder, viz., type $2 \mathrm{DM}$, accounts for 90$95 \%$ of the total DM patients. ${ }^{3}$ The mechanism of type 2 DM is well known, stemming from the ineffective use of insulin in the body or insufficient insulin production in the pancreas of patients.

Inhibition of glucose- and insulin-based enzymes is the main strategy for the therapeutic treatment of type 2 diabetes. The former attempts to reduce gut glucose absorption by controlling postprandial hyperglycemia by mitigating the activity of glucosidases; meanwhile, the latter involves insulin signaling 

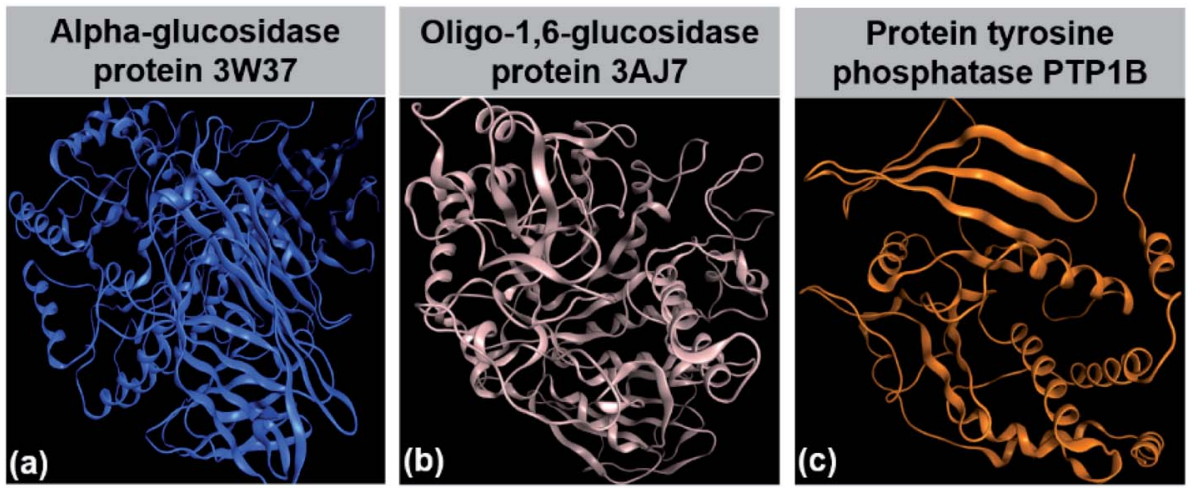

Fig. 1 (a) $\alpha$-Glucosidase protein 3W37. (b) Oligo-1,6-glucosidase protein 3W37. (c) Protein tyrosine phosphatase 1B PTP1B

regulation by compensating defects in insulin secretion and insulin action. ${ }^{4}$ Firstly, $\alpha$-glucosidase, an exoenzyme found in animals, plants, bacteria, and fungi, breaks down starch and disaccharides to glucose. A study suggested that this enzyme can only yield monosaccharides by catalysing the hydrolysis of $\alpha-(1 \rightarrow 4)$ and $\alpha-(1 \rightarrow 6)$ bonds, ${ }^{5}$ confirming the sources of $\alpha-$ glucosidase from sugar beet seeds. ${ }^{6}$ Data on the $\alpha$-glucosidase crystal structure can be found at the Worldwide Protein Data Bank under entry PDB-3W37 (DOI: 10.2210/pdb3W37/pdb). Secondly, oligo-1,6-glucosidase, also known as isomaltase, is a debranching endoenzyme, hydrolysing only the $\alpha-1,6$ linkage in starch and glycogen to produce sugars with an $\alpha$-configuration. ${ }^{7}$ Besides some bacterial species such as Bacillus cereus, oligo-1,6-glucosidase is present mainly in the animal kingdom. ${ }^{8}$ In humans, it can be found on the small intestine brush border. ${ }^{9}$ Protein structural data of isomaltase can be download directly from the Worldwide Protein Data Bank database under entry PDB-3AJ7 (DOI: 10.2210/pdb3AJ7/pdb). Thirdly, protein tyrosine phosphatase 1B (PTP1B) is a major glucosehomeostasis and energy-metabolism regulator, and thus regarded as an attractive drug target for therapeutic intervention in type 2 diabetes and obesity. ${ }^{10}$ It blocks insulin receptor substrate-1 and dephosphorylate phosphotyrosine residues, thus causing insulin insensitivity or even cut-off of intracellular insulin signaling. ${ }^{11}$ Conversely, regarding the leptin signaling pathway, this protein binds and dephosphorylates leptin receptor Janus kinase 2 (JAK2), thereby resulting in the malfunctioning of energy balance. ${ }^{11}$ The PTP1B structure has already been well determined and its information is available for public reference at UniProtKB, archived under entry ID: UniProtKB-A0A0U1XP67. Therefore, 3W37, 3AJ7, and PTP1B (Fig. 1) are considered highly promising drug targets for the effective treatment of type 2 diabetes. In principle, multiple inhibition of the protein tyrosine phosphatase 1B (PTP1B) and glycoside hydrolase proteins (3W37 and $\mathbf{3 A J} 7$ ) is a promising strategy to suppress hyperglycemia and improve insulin sensitisation simultaneously.

Natural products are considered highly valuable for drug discovery due to their diverse structures and biological activities. In particular, triterpenes belong to a large group of naturally occurring compounds derived from the precursor squalene..$^{12}$ Dammarane is a tetracyclic triterpene, existing as the main components in dammar resin ${ }^{13,14}$ and ginseng. ${ }^{15}$ The basic skeleton of dammarane is comprised of a tetracyclic moiety and a side chain at C-17..$^{16,17}$ Recently, the number of publications and patents on the anti-diabetic activity of dammarane triterpenoids has increased rapidly. ${ }^{18-21}$ Besides, clinical trials and animal model experiments have proven the ability of dammarane triterpenoids to prevent and treat diabetes by lowering the blood glucose, regulating lipid metabolism, and increasing insulin sensitivity. ${ }^{22}$ Thus, considering the advantage of the diversity and novelty of the dammarane structure, a series of chemical modifications of leading compounds has been achieved. Consequently, many synthetic dammarane derivatives have been reported to improve hyperglycemia, glucose tolerance and $\alpha$-glucosidase inhibition. ${ }^{22-25}$ Dipterocarpol,

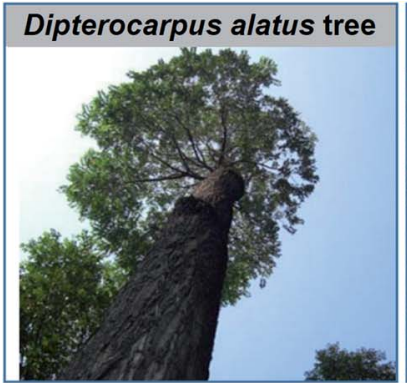

(a)

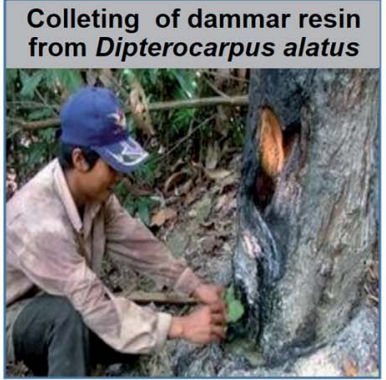

(b)

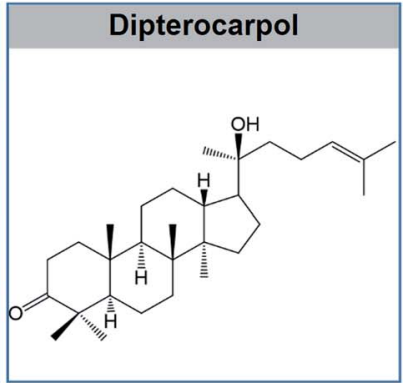

(c)

Fig. 2 (a) Dipterocarpus alatus tree. (b) Collection of dammar resin from Dipterocarpus alatus. (c) Structure of dipterocarpol. 
a dammarane-type triterpenoid, is the main metabolite of the resin from Dipterocarpus alatus (Dipterocarpaceae), a tropical plant growing in countries in South East Asia such as Cambodia, Laos, Thailand, the Philippines and Vietnam ${ }^{26}$ (Fig. 2). It was reported that dipterocarpol and its derivatives exhibit antituberculosis, ${ }^{27}$ antimicrobial, ${ }^{28}$ hepatoprotective, ${ }^{29}$ and cytotoxic activity. ${ }^{30}$

In this work, two different approaches to modify the structure of dipterocarpol are proposed based on their advantages reported in the literature. The modification of the ring at C-3 of dipterocarpol to obtain oxime and its acyl derivatives (Fig. 3a) was designed. The activating group ketone at C-3 can be easily converted to oxime, followed by $O$-acylation to obtain acyloxyimino compounds $(-\mathrm{CR}=\mathrm{N}-\mathrm{O}-\mathrm{COOR})$. This type of compound (containing acyloxyimino functional groups) is known to exhibit important biological activities, e.g., antiviral, anticancer and antidiabetes. ${ }^{31}$ According to our knowledge, several studies on the synthesis of oxime esters derived from lupane or oleane triterpenoids have been carried out; ${ }^{31}$ nevertheless, similar transformation regarding dammarane triterpenoids has not reported to date. Another modification was at the side chain, focusing on the formation of a lactone ring, instead of a long-chain linear of dipterocarpol (Fig. 3b). Previous studies showed that the location of a lactone ring at $\mathrm{C}-17$ of some dammarane derivatives helps promote the antihyperglycemic effects significantly. ${ }^{\mathbf{2 0 , 2 5 , 3 2}}$ Besides, a series of ester derivatives at C-3 was also introduced.

In silico research has been developed extensively in an attempt to accurately predict molecular behaviour at the biological scale. In particular, molecular dynamics gives a view of the dynamic "evolution" of atoms and molecules in a system, whose trajectories are determined by numerically solving Newton's equations of motion for a system of interacting particles. This technique is widely known as one of the most powerful computer-aided tools for pharmacophore development and drug design. However, its downside of long computation times, high cost and significant power consumption highly limit its applicability. Conversely, although successfully complementing the dynamics-related disadvantage by introducing a view of static inhibitory stability, the molecular docking technique is based on a precursor condition that the ligands are already placed within a certain radius to the targeted protein, and thus there is a lack of information on their preceding transport in or interaction with plasmatic components in biological media. Accordingly, a strategy based on the combination of the static docking technique and molecular dynamics has been proposed in an attempt to retrieve

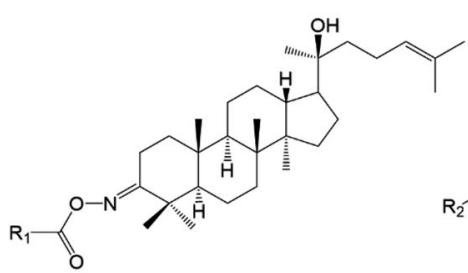

(a)

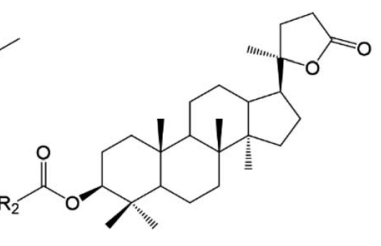

(b)
Fig. 3 Designed structures of dipterocarpol derivatives. meaningful models for ligand-protein inhibition. ${ }^{33-35}$ Nevertheless, the cost of its implementation is still considered high in low-based research institutes. QSARIS, ${ }^{36}$ which was released by SciVision Biotech Inc., is a leading-edge database system for predicting the physiochemical properties of lead compounds in drug and agrochemical research. This software promises sufficient compensation for the information lost in the pre-docking presumption. Therefore, a QSARIS-docking combination can be a potential approach for the prediction of ligand-protein inhibition, in general.

Herein, both experimental and theoretical studies were conducted. The isolation of dipterocarpol and semi-synthesis of its twenty derivatives were carried out on Dipterocarpus alatus from Vietnam. Their $\alpha$-glucosidase inhibition was investigated by enzyme assays. The experimental evidence collected was used to retrieve a comprehensive theoretical model framed by molecular docking simulation coupled with QSARIS-based analysis. The experiment-theory retrieval was applied to all the diabetes-related protein structures.

\section{Methodology}

\subsection{Experiment}

2.1.1. Materials and characterisation. Reagents and solvents were purchased from commercial suppliers and used without further purification. Column chromatography was performed using silica gel from Merck (Kieselgel 60, 230-400 mesh). Analytical thin-layer chromatography (TLC) was carried out on pre-coated silica gel $60 \mathrm{~F}_{254}$ sheets (0.25 mm, Merck). Nuclear magnetic resonance (NMR) spectroscopic data was acquired on a Bruker Advance III spectrometer (Switzerland) with the chemical shift in ppm using tetramethylsilane (TMS) as the internal standard. Electrospray ionization mass spectrometry (ESI-MS) was performed on an Agilent 1100 mass spectrometer from the USA. Fourier-transform infrared (FT-IR) spectroscopy was performed on a PerkinElmer Spectrum Two (UK). High-resolution electrospray ionization mass spectra (HRESI-MS) were recorded on a SCEIX X500R QTOF (USA). TLC spots were visually observed under UV light (254 and $365 \mathrm{~nm}$ ) and by dipping in vanillin/ $\mathrm{H}_{2} \mathrm{SO}_{4}$ reagent followed by heating.

\subsubsection{Isolation and synthesis}

Isolation of dipterocarpol (compound 1) from Dipterocarpus alatus. The resin of Dipterocarpus alatus $(4.0 \mathrm{~kg})$ was collected in Dai Thach commune, Dai Loc district, Quang Nam province, Vietnam in April, 2018. The plant sample was identified taxonomically by Mst. Nguyen The Anh, Institute of Chemistry, Vietnam Academy of Science and Technology, Hanoi, Vietnam. Water (2 L) was added to the resin, and successively extracted with $n$-hexane $(4 \times 2 \mathrm{~L})$ and $\mathrm{CH}_{2} \mathrm{Cl}_{2}(3 \times 2 \mathrm{~L})$ at room temperature. The extracts were combined and evaporated under reduced pressure to obtain the $n$-hexane and dichloromethane extracts, respectively. The dichloromethane extract was dipped in $\mathrm{MeOH}$ ( $2 \mathrm{~L}$, for $5 \mathrm{~h}$ ). Finally, the solvent of the $\mathrm{MeOH}$ extract was removed under reduced pressure to obtain a white solid, which was recrystalised in EtOH to yield dipterocarpol (80 g).

The synthesis of the oxime esters is summarised in Scheme 1. 
Synthesis of oxime 2. A solution of compound 1 (500 mg, 1.13 $\mathrm{mmol})$, hydroxylamine hydrochloride (142 $\mathrm{mg}, 2.03 \mathrm{mmol}$ ) and triethylamine $(0.1 \mathrm{~mL})$ in ethanol $(3 \mathrm{~mL})$ was stirred at room temperature for $24 \mathrm{~h}$. The solvent was removed under vacuum and water $(20 \mathrm{~mL})$ was added to the residue, followed by extraction with $\mathrm{CH}_{2} \mathrm{Cl}_{2}(3 \times 20 \mathrm{~mL})$. The $\mathrm{CH}_{2} \mathrm{Cl}_{2}$ extracts were combined, dried over anhydrous $\mathrm{Na}_{2} \mathrm{SO}_{4}$, and the solvent evaporated to obtain a white solid. The solid was purified by silica gel column chromatography ( $n$-hexane/EtOAc $9: 1$ ) to obtain compound 2 (yield: $430 \mathrm{mg}, 83.0 \%$ ).

General procedures for the synthesis of oxime esters $3 a-3 i$. To a solution of compound 2 ( $40 \mathrm{mg}, 0.087 \mathrm{mmol}, 1 \mathrm{eq}$.), DCC (36 mg, $0.174 \mathrm{mmol}, 2$ eq.) and DMAP $(7.42 \mathrm{mg}, 0.06 \mathrm{mmol})$ in $\mathrm{CH}_{2} \mathrm{Cl}_{2}(5 \mathrm{~mL})$ were added with carboxylic acid $(0.174 \mathrm{mmol}, 2$ eq.). The reaction mixture was stirred at room temperature for $5 \mathrm{~h}$, and then filtered to separate the solution from the solid. The solution was evaporated under vacuum, followed by the addition of water to yield a white solid, which was purified by silica gel column chromatography ( $n$-hexane/EtOAc) to attain compounds $3 \mathbf{a}-3 \mathbf{i}$ ( $48-80 \%$ yield).

Synthesis of oxime ester $3 \boldsymbol{k}$. To a solution of compound 2 (40 $\mathrm{mg}, 0.087 \mathrm{mmol})$ in pyridine $(1 \mathrm{~mL})$, acetic anhydride $(0.3$ $\mathrm{mL}$ ) was added. The reaction mixture was stirred at room temperature for $24 \mathrm{~h}$. The solvent was removed under reduced pressure to obtain a white residue. The residue was purified on a silica gel column ( $n$-hexane/EtOAc $87: 13$ ) to yield $35 \mathrm{mg} \mathbf{3 k}$ (80.6\%).

General procedures for the synthesis of oxime esters $3 \mathbf{l}$ and $\mathbf{3 m}$. To a solution of compound $2(40 \mathrm{mg}, 0.087 \mathrm{mmol})$ in $\mathrm{CH}_{2} \mathrm{Cl}_{2}$ (2.5 mL), valeryl chloride $(0.015 \mathrm{~mL}, 1.25 \mathrm{eq}$. for compound $3 \mathrm{l})$ or isobutyryl chloride ( $0.013 \mathrm{~mL}, 1.25$ eq. for compound $3 \mathrm{~m}$ ) was added. The reaction mixture was stirred at room temperature for $19 \mathrm{~h}$, and then the solvent was evaporated under reduced pressure. The white crude product was purified by silica gel column chromatography ( $n$-hexane/EtOAc $9: 1$ ) to afford compound 31 (39 mg, 83\%) or compound $3 \mathbf{m ~ ( 3 8 ~ m g , ~}$ $82 \%)$.

The synthesis of the lactone derivatives is summarised in Scheme 2.

Synthesis of compound 4. A mixture of $\mathrm{CrO}_{3}(2.45 \mathrm{~g}$, $16.2 \mathrm{mmol}, 2.8$ eq.) in $\mathrm{CH}_{3} \mathrm{COOH}(5 \mathrm{~mL})$ and $\mathrm{H}_{2} \mathrm{O}(3 \mathrm{~mL})$ was added dropwise to a solution of compound 1 (2.5 g, $5.65 \mathrm{mmol}$, 1 eq.) in $\mathrm{CH}_{3} \mathrm{COOH}(20 \mathrm{~mL})$ at $5{ }^{\circ} \mathrm{C}$. The reaction mixture was allowed to warm up to room temperature and further stirred for $30 \mathrm{~min}$. A solution of $10 \% \mathrm{NaCl}(50 \mathrm{~mL})$ was added to the reaction mixture, followed by extraction with EtOAc $(3 \times 50$ $\mathrm{mL}$ ). The extract was evaporated under vacuum to yield a white solid, which was purified by silica gel column chromatography ( $n$-hexane/acetone $9: 1)$ to yield compound 4 (2.0 g, $4 \mathrm{mmol}$, $85.5 \%)$.

Synthesis of compound 5. To a solution of compound 4 (700 mg, $1.7 \mathrm{mmol}, 1$ eq.) in $\mathrm{MeOH}(7 \mathrm{~mL})$ at $5{ }^{\circ} \mathrm{C}, \mathrm{NaBH}_{4}$ (260 mg, $6.84 \mathrm{mmol}, 4$ eq.) was added slowly. The reaction mixture was allowed to warm up to room temperature and further stirred for $20 \mathrm{~min}$. Acetone $(5 \mathrm{~mL})$ was added, and the reaction mixture was continuously stirred for an additional 5 min. Cold water $(5 \mathrm{~mL})$ was added to the reaction, followed by evaporation. Water $(30 \mathrm{~mL})$ was added to the residue, and then extracted with EtOAc $(3 \times 30 \mathrm{~mL})$. The solvent was removed to give a white powder, which was purified by silica gel column chromatography ( $n$-hexane/EtOAc $9: 1$ ) to yield compound 5 (520 mg, 74\%).

Synthesis of compound $\mathbf{6 a}$. To a solution of compound $\mathbf{5}$ $(20 \mathrm{mg}, 0.048 \mathrm{mmol})$ in pyridine $(1 \mathrm{~mL})$, acetic anhydride $(0.3$ $\mathrm{mL}$ ) was added. The reaction mixture was stirred at room temperature for $24 \mathrm{~h}$. The solvent was removed under reduced pressure to obtain a white residue. The residue was purified by

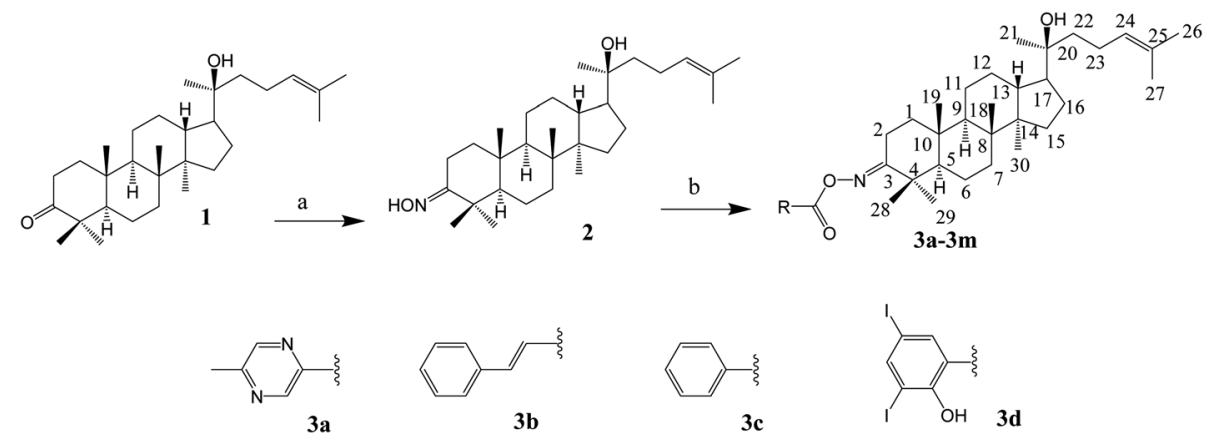

$\mathrm{R}$

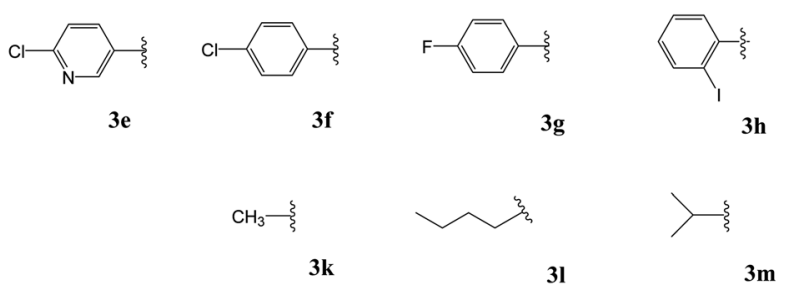

Scheme 1 Synthesis of oxime ester derivatives at $\mathrm{C}-3$ of dipterocarpol. (a) $\mathrm{NH}_{2} \mathrm{OH} \cdot \mathrm{HCl}, \mathrm{TEA}, \mathrm{C}_{2} \mathrm{H}_{5} \mathrm{OH}, 24 \mathrm{~h}, \mathrm{rt}$ (83\%). (b) Synthesis of 3a-3i: DCC, DMAP, $\mathrm{RCOOH}, \mathrm{CH}_{2} \mathrm{Cl}_{2}, 5 \mathrm{~h}$, rt (48-80\%); 3k: pyridine, $\mathrm{Ac}_{2} \mathrm{O}, 24 \mathrm{~h}$, rt (81\%); 3l, 3m: $\mathrm{RCOCl}, \mathrm{CH}_{2} \mathrm{Cl}_{2}, 19 \mathrm{~h}$, rt (80-83\%). 


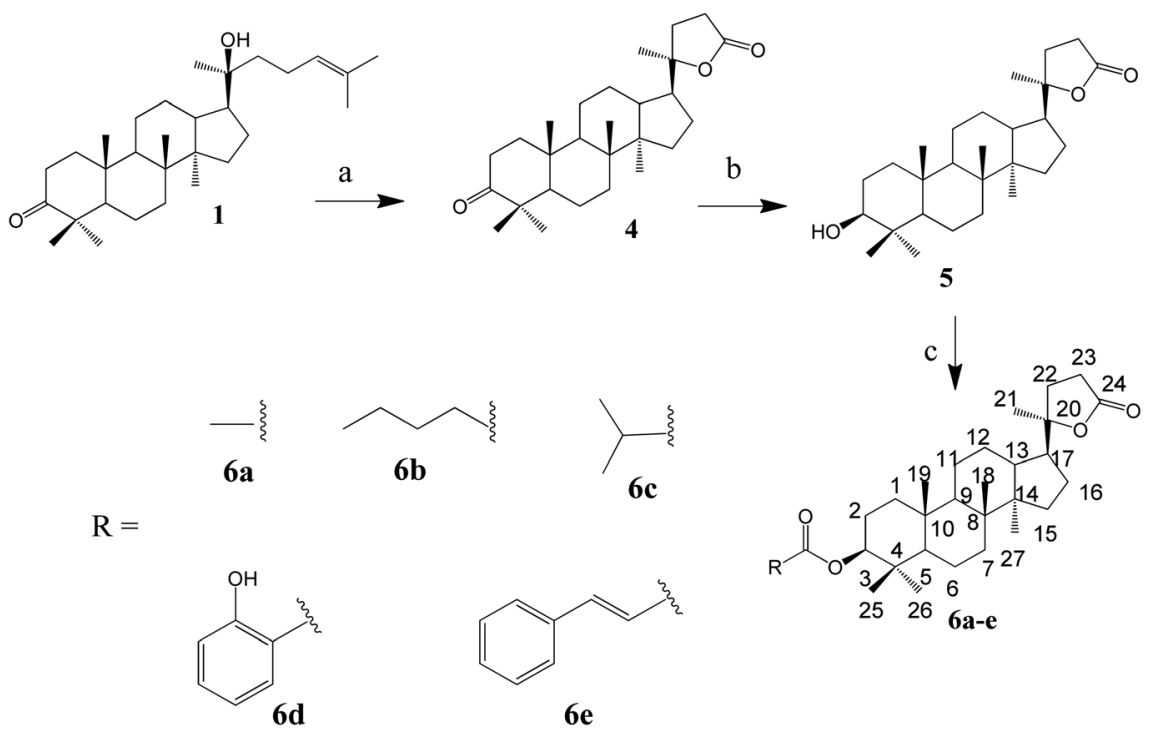

Scheme 2 Synthesis of lactone derivatives of dipterocarpol. (a) $\mathrm{CrO}_{3}, \mathrm{CH}_{3} \mathrm{COOH}, \mathrm{H}_{2} \mathrm{O}, 40 \mathrm{~min}$, rt (85.5\%). (b) $\mathrm{NaBH}, \mathrm{MeOH}, 20 \mathrm{~min}, \mathrm{rt}(74 \%)$. (c) Synthesis of $6 \mathrm{a}: \mathrm{Ac}_{2} \mathrm{O}$, pyridine, $24 \mathrm{~h}$, rt (73\%); $6 \mathrm{~b}$ and $6 \mathrm{c}: \mathrm{RCOCl}, \mathrm{CH}_{2} \mathrm{Cl}_{2}, 20 \mathrm{~h}$, rt (60-75\%); $6 \mathrm{~d}$ and $6 \mathrm{e}: \mathrm{RCOOH}, \mathrm{DCC}, \mathrm{DMAP}, \mathrm{CH} \mathrm{Cl}_{2}, \mathrm{rt}, 48 \mathrm{~h}$ for $6 \mathrm{~d}(66 \%)$ or reflux, $28 \mathrm{~h}$ for $6 \mathrm{e}(61 \%)$.

silica gel column chromatography $(n$-hexane/EtOAc $9: 1)$ to give compound $6 \mathbf{a}(16 \mathrm{mg}, 73 \%)$.

General procedure for the synthesis of compounds $\boldsymbol{6} \boldsymbol{b}$ and $\boldsymbol{6} \boldsymbol{c}$. To a solution of compound 5 (20 mg, $0.048 \mathrm{mmol}, 1$ eq.) in $\mathrm{CH}_{2} \mathrm{Cl}_{2}$ $(2.5 \mathrm{~mL})$, valeryl chloride $(0.015 \mathrm{~mL}, 2.5$ eq., for compound $\mathbf{6 b})$ or isobutyryl chloride $(0.013 \mathrm{~mL}, 2.5$ eq., for compound $\mathbf{6 c})$ was added. The reaction mixture was stirred at room temperature for $20 \mathrm{~h}$, and then the solvent evaporated under reduced pressure. The obtained white crude product was purified by Sephadex LH-20 column chromatography ( $n$-hexane $/ \mathrm{CH}_{2} \mathrm{Cl}_{2} /$ $\mathrm{MeOH} 1 / 1 / 2)$ to obtain the respective compound $6 \mathbf{b}(18 \mathrm{mg}$, $75 \%)$ or compound $6 \mathbf{c}(14 \mathrm{mg}, 60 \%)$.

General procedures for the synthesis of compounds $\mathbf{6 \boldsymbol { d }}$ and $\mathbf{6 e}$. To a solution of compound $5(20 \mathrm{mg}, 0.048 \mathrm{mmol}, 1 \mathrm{eq}$.$) , DCC$ (20 mg, $0.096 \mathrm{mmol}, 2.0$ eq.) and DMAP (6 mg, $0.048 \mathrm{mmol}, 1.0$ eq.) in $5 \mathrm{~mL} \mathrm{CH}_{2} \mathrm{Cl}_{2}$ were added with carboxylic acid (2 eq., 0.096 $\mathrm{mmol})$. The reaction mixture was stirred for $48 \mathrm{~h}$ at room temperature (for compound 6d) or refluxed for $28 \mathrm{~h}$ (for compound $\mathbf{6 e}$ ). The white precipitate was filtered. The remaining solution was evaporated under vacuum, and water was added and extracted with EtOAc $(3 \times 15 \mathrm{~mL})$. The solvent was removed from the extract, and the remaining residue was subjected to a silica gel column ( $n$-hexane/EtOAc) to obtain compound $6 \mathbf{d}(17 \mathrm{mg}, 66 \%)$ or $\mathbf{6 e}(16 \mathrm{mg}, 61 \%)$.

2.1.3. $\alpha$-Glucosidase inhibitory assay. The bioassay was carried out on 96 well-plates ( $200 \mu \mathrm{L}$ per well). A solution of the test samples in $10 \%$ dimethyl sulfoxide (DMSO) was diluted further to concentrations of 1024, 256, 64, 16, 4, and $1 \mu \mathrm{g} \mathrm{mL} \mathrm{m}^{-1}$. Each concentration of the test samples $(10 \mu \mathrm{L})$ was incubated at $37^{\circ} \mathrm{C}$ with phosphate buffer $100 \mathrm{mM}(\mathrm{pH} 6.8,40 \mu \mathrm{L}), \alpha$-glucosidase $0.4 \mathrm{U} \mathrm{mL}^{-1}(25 \mu \mathrm{L})$, and $p$-nitrophenyl $\alpha$-D-glucopyranoside $2.5 \mathrm{mM}(25 \mu \mathrm{L})$. The reaction was stopped after $30 \mathrm{~min}$ by adding $100 \mu \mathrm{L}$ of $0.1 \mathrm{M} \mathrm{Na}_{2} \mathrm{CO}_{3}$. The enzyme activities were measured based on the absorbance at $\lambda_{\max } 405 \mathrm{~nm}$ of the test samples. The control assay was prepared using the same procedure, except the test samples were replaced with distilled water; meanwhile, the activity of the reference was tested by replacing the sample with acarbose. ${ }^{37}$ Percentage inhibition was calculated as follows: ${ }^{38}$

$$
\begin{aligned}
\alpha \text {-Glucosidase inhibition }(\%)= & {\left[\mathrm{Abs}_{(\mathrm{control})}-\mathrm{Abs}_{(\text {(sample })}\right] } \\
& / \mathrm{Abs}_{(\mathrm{control})} \times 100 \%
\end{aligned}
$$

\subsection{Computation}

2.2.1. Molecular docking simulation. The MOE 2015.10 software was used for the molecular docking simulation. Its requisite input includes structural information of the docking participants, i.e., 21 Dipterocarpus alatus-derived ligands and 3 diabetes-related protein structures. The former is dipterocarpol (1), 13 oxime esters ( $\mathbf{2}$ and $\mathbf{3 a - 3 m}$ ), and 7 lactones $(\mathbf{4}, \mathbf{5}$, and $\mathbf{6 a -}$ 6e) and the latter is glycoside-hydrolase proteins (3W37 and 3AJ7) and protein tyrosine phosphatase 1B (PTP1B). The formation of each ligand-protein inhibitory structure was primarily evaluated using a variety of output parameters, e.g., docking score (DS) energy, root-mean-square deviation (RMSD), interaction types, and distance between ligands and proteins. A typical docking procedure followed three steps. ${ }^{39-42}$

Pre-docking preparation. Structural information of three proteins 3W37, 3AJ7, and PTP1B was obtained from the Worldwide Protein Data Bank and UniProtKB: $\alpha$-glucosidase protein 3W37 (DOI: 10.2210/pdb3W37/pdb), oligo-1,6glucosidase protein 3AJ7 (DOI: 10.2210/pdb3AJ7/pdb), and tyrosine phosphatase protein 1B PTP1B (entry: UniProtKBA0A0U1XP67). Sequence Editor in the MOE 2015.10 program was used to remove the water molecules in the protein structure. The Quickprep tool was then used to prepare the structure 
of the proteins and their 3D protonation, configured as: tethered ligand-receptor with the strength of 5000 and refinement of $0.0001 \mathrm{kcal} \mathrm{mol}^{-1} \AA^{-1}$. The active zones of the proteins were determined based on the possible interactability between their amino acids and the inhibitory ligands within a radius of $4.5 \AA$. The protein structural data obtained was saved in the *.pdb format. The ligands were under energy optimisation via Conj Grad and Termination. The configuration was set with an energy change of $0.0001 \mathrm{kcal} \mathrm{mol}^{-1}$; maximum number of interactions of 1000; and a modified Gasteiger-Huckel charge.

Docking investigation. After input preparation, intermolecular interaction simulation was performed on MOE 2015.10 and the complex structures were saved in the *.sdf format. The docking simulation parameters were configured as follows: the number of poses retained for further inhibition analysis $=10$; the maximum number of solutions per iteration $=1000$; and the maximum number of solutions per fragmentation $=200$.

Post-docking analysis. The docking score (DS) predicts the binding affinity of ligands and their targeted protein in the sitesite distance. The conformation of the docked complexes was visualised on $2 \mathrm{D}$ and $3 \mathrm{D}$ planes. The interactions formed in the active sites between the ligands and amino acids in the site-site distance of their targeted protein were also analysed. They include hydrogen bonds, ion bonds, arene-arene $(\tau-\pi)$, cationarene (cations $-\pi$ ), and van der Waals interactions detected during the simulation. In addition, root-mean-square deviation (RMSD) calculated provides information on the rigidity of the conformational binding of the docked complexes.

2.2.2. Physicochemical and pharmaceutical compatibility. The docking parameters including $\mathrm{DS}_{\text {average }}\left(\mathrm{kcal} \mathrm{mol}^{-1}\right)$, molecular mass (Da), polarizability $\left(\AA^{3}\right)$ and volume or size $(\AA)$, and dispersion coefficients $(\log P$ and $\log S)$ were achieved with the Gasteiger-Marsili method using the QSARIS system. ${ }^{\mathbf{4 3}}$ They were then pre-screened in an attempt to evaluate their oral pharmacological compatibility. This was based on Lipinski's rule of five, a well-known set of indicators to predict drug-likeness. ${ }^{\mathbf{4 4}}$ According to Lipinski's criteria, a good membranepermeable molecule should satisfy the following requirements: (1) molecular mass of $<500 \mathrm{Da}$; (2) no more than 5 groups for hydrogen bonds; (3) no more than 10 groups receiving hydrogen bonds; and (4) $\log P$ value of less than +5 $(\log P<5){ }^{45,46}$

\section{Results and discussion}

\subsection{Experimentation}

The synthesis of the oxime ester and lactone derivatives from dipterocarpol is schematically described in Schemes 1 and 2, respectively. Firstly, dipterocarpol was allowed to react with hydroxylamine hydrochloride/triethylamine in ethanol for $24 \mathrm{~h}$ to obtain oxime 2 in $83 \%$ yield. This product was then acylated with carboxylic acids (3a-3i), chloride acids ( $\mathbf{3} \mathbf{l}$ and $\mathbf{3 m}$ ) or acetic anhydride/pyridine (3k) to afford a series of oxime ester $\mathbf{3 a - 3 m}$ (Scheme 1). It was noted that in the case of anhydride or chloride acid, if the reaction was prolonged for more than $24 \mathrm{~h}$, diester derivatives at C-3 (major) and C-20 (minor) were formed.
To the best of our knowledge, there was only one study reported on the oxidative cleavage at the double bond (C-24 and C25) of dipterocarpol. The authors used ozone $/ \mathrm{CH}_{2} \mathrm{Cl}_{2}$ or ozone/ $\mathrm{MeOH}$ to obtain hemiacetal or acetal, respectively. ${ }^{21}$ In our case, other oxidative cleavage reagents were utilised such as $\mathrm{CrO}_{3}$, $\mathrm{SeO}_{2}, \mathrm{KMnO}_{4}$. Consequently, the use of $\mathrm{CrO}_{3} / \mathrm{CH}_{3} \mathrm{COOH}$ (Jones oxidation) afforded the lactone products (compound 4) under mild conditions (room temperature, $40 \mathrm{~min}$ ) in good yield $(85.5 \%)$. Other oxidative reagents gave several unwanted products, which could not be purified after the reaction. Under Jones oxidation, the aldehyde formed at C-24 was further oxidised to carboxylic acid, which continuously underwent an intramolecular reaction with the hydroxyl group at C-20 to form lactone 4 in acidic condition during oxidation. The reduction of the ketone group at $\mathrm{C}-3$ of compound 4 to hydroxyl group was carried out with $\mathrm{NaBH}_{4}$ in $\mathrm{MeOH}$ for 20 min. Compound 5 was obtained in $74 \%$ yield. Due to the steric hindrance in the $\beta$-site of the A-ring of 4 , the $\mathrm{BH}_{4}{ }^{-}$anion can only approach the C-3keto group from the $\alpha$-site, and thus the $3 \beta$-hydroxyl product $\mathbf{5}$ is the major product. Compound $\mathbf{5}$ was the only product obtained after column chromatography of the reduction mixture. Further, $\mathbf{5}$ was reacted with anhydric acid/pyridine to produce $\mathbf{6 a}$, with chloride acid to give $\mathbf{6 b}$ and $\mathbf{6 c}$, and with carboxylic acid/DCC/DMAP to obtain $\mathbf{6 d}$ and $\mathbf{6 e}$ in good yields.

\subsection{Characteristics}

\subsubsection{Spectroscopic data}

Compound 1 (dipterocarpol). White needles, $\mathrm{mp}$ 128.4130.3 ${ }^{\circ} \mathrm{C} ; \mathrm{C}_{30} \mathrm{H}_{50} \mathrm{O}_{2}$, ESI-MS (m/z): $443.2[\mathrm{M}+\mathrm{H}]^{+}, 425.2[\mathrm{M}+\mathrm{H}-$ $\left.\mathrm{H}_{2} \mathrm{O}\right]^{+} ;{ }^{1} \mathrm{H}$ NMR (500 MHz, $\left.\mathrm{CDCl}_{3}, \delta_{\mathrm{H}}, \mathrm{ppm}\right),(J, \mathrm{~Hz}): 5.13-5.10$ (1H, m), 2.53-2.39 (2H, m), 2.08-2.02 (2H, m), 1.94-1.89 (1H, $\mathrm{m}), 1.85-1.83(1 \mathrm{H}, \mathrm{m}), 1.76-1.71(2 \mathrm{H}, \mathrm{m}), 1.69(3 \mathrm{H}, \mathrm{s}), 1.62(3 \mathrm{H}$, s), 1.59-1.23 (16H), $1.15(3 \mathrm{H}, \mathrm{s}), 1.08(3 \mathrm{H}, \mathrm{s}), 1.03(3 \mathrm{H}, \mathrm{s}), 1.00$ $(3 \mathrm{H}, \mathrm{s}), 0.94(3 \mathrm{H}, \mathrm{s}), 0.89(3 \mathrm{H}, \mathrm{s}) .{ }^{13} \mathrm{C} \mathrm{NMR}\left(125 \mathrm{MHz}, \mathrm{CDCl}_{3}, \delta_{\mathrm{C}}\right.$, ppm): 218.08, 131.63, 124.69, 75.36, 55.38, 50.26, 50.02, 49.84, 47.42 , 42.40, 40.47, 40.29, 39.89, 36.84, 34.56, 34.11, 31.15, $27.53,26.71,25.72,25.48,24.80,22.56,22.04,21.00,19.66$, $17.70,16.35,16.00,15.22$.

Compound 2 [20(S)-hydroxydammar-24-ene-3-oxime]. White amorphous powder, $\mathrm{C}_{30} \mathrm{H}_{51} \mathrm{NO}_{2}$, ESI-MS $(\mathrm{m} / \mathrm{z}): 458.3[\mathrm{M}+\mathrm{H}]^{+}$, $440.2\left[\mathrm{M}+\mathrm{H}-\mathrm{H}_{2} \mathrm{O}\right]^{+} ;{ }^{1} \mathrm{H}-\mathrm{NMR}\left(500 \mathrm{MHz}, \mathrm{CDCl}_{3}, \delta_{\mathrm{H}}, \mathrm{ppm}\right),(J$, $\mathrm{Hz}): 5.12(\mathrm{t}, 1 \mathrm{H}, J=7.0), 2.97-2.92(1 \mathrm{H}, \mathrm{m}), 2.31-2.25(1 \mathrm{H}, \mathrm{m})$, 2.07-2.02 (2H, m), 1.84-1.78 (2H, m), 1.75-1.72 (2H, m), 1.68 $(3 \mathrm{H}, \mathrm{s}), 1.62(3 \mathrm{H}, \mathrm{s}), 1.14(3 \mathrm{H}, \mathrm{s}), 1.14(3 \mathrm{H}, \mathrm{s}), 1.05(3 \mathrm{H}, \mathrm{s}), 0.99$ $(3 \mathrm{H}, \mathrm{s}), 0.94(3 \mathrm{H}, \mathrm{s}), 0.86(3 \mathrm{H}, \mathrm{s}) .{ }^{13} \mathrm{C}-\mathrm{NMR}\left(125 \mathrm{MHz}, \mathrm{CDCl}_{3}, \delta_{\mathrm{C}}\right.$, ppm): 167.40, 131.62, 124.74, 75.41, 56.03, 50.31, 50.30, 49.87, $42.37,40.53,40.43,40.37,39.08,37.20,34.86,31.16,27.56$, $27.41,25.73,25.47,24.81,22.88,22.58,21.84,19.07,17.72$, 17.03, 16.37, 15.89, 15.41.

Compound $3 \boldsymbol{a} \quad\{20($ S)-hydroxy dammar-24-ene-3-oximyl-[5methyl-3,6-diazine-1-carboxylate]\}. White amorphous powder, $\mathrm{C}_{36} \mathrm{H}_{55} \mathrm{~N}_{3} \mathrm{O}_{3}$, HR-ESI-MS $(\mathrm{m} / \mathrm{z})$ : $600.4285[\mathrm{M}+\mathrm{Na}]^{+}$(calculated for $\mathrm{C}_{36} \mathrm{H}_{55} \mathrm{~N}_{3} \mathrm{O}_{3} \mathrm{Na}$ 600.4245); ${ }^{1} \mathrm{H}-\mathrm{NMR}\left(500 \mathrm{MHz}, \mathrm{CDCl}_{3}, \delta_{\mathrm{H}}\right.$, ppm), (J, Hz): 9.18 (1H, d, $J=1.5), 8.58(1 \mathrm{H}, \mathrm{d}, J=1.5), 5.13-5.10$ $(1 \mathrm{H}, \mathrm{m}), 2.98-2.93(1 \mathrm{H}, \mathrm{m}), 2.67(3 \mathrm{H}, \mathrm{s}), 2.61-2.54(1 \mathrm{H}, \mathrm{m}), 2.07-$ $2.02(2 \mathrm{H}, \mathrm{m}), 1.68(3 \mathrm{H}, \mathrm{s}), 1.56(3 \mathrm{H}, \mathrm{s}), 1.33(3 \mathrm{H}, \mathrm{s}), 1.21(3 \mathrm{H}, \mathrm{s})$, 
1.14 (3H, s), 1.00 (3H, s), 0.97 (3H, s), 0.88 (3H, s). ${ }^{13} \mathrm{C}-\mathrm{NMR}(125$ $\left.\mathrm{MHz}, \mathrm{CDCl}_{3}, \delta_{\mathrm{C}}, \mathrm{ppm}\right): 177.84,162.08,157.72,145.27,144.33$, 140.69, 131.65, 124.71, 75.38, 55.93, 50.28, 49.89, 42.35, 41.89, $40.47,40.42,39.47,37.17,34.73,33.98,31.15,27.51,27.48$, $25.74,25.51,24.81,22.70,22.58,21.97,21.94,20.18,19.03$, 17.71, 16.36, 16.21, 15.37 .

Compound $3 \boldsymbol{b}$ [20(S)-hydroxydammar-24-ene-3-oximyl cinnamate]. White amorphous powder, $\mathrm{C}_{39} \mathrm{H}_{57} \mathrm{NO}_{3}$, HR-ESI-MS $(\mathrm{m} / \mathrm{z})$ : $610.4251[\mathrm{M}+\mathrm{Na}]^{+}$(calculated for $\mathrm{C}_{39} \mathrm{H}_{57} \mathrm{NO}_{3} \mathrm{Na}$ 610.4236), ESIMS $(m / z): 588.1[\mathrm{M}+\mathrm{H}]^{+} ;{ }^{1} \mathrm{H}-\mathrm{NMR}\left(500 \mathrm{MHz}, \mathrm{CDCl}_{3}, \delta_{\mathrm{H}}, \mathrm{ppm}\right)$, $(J, \mathrm{~Hz}): 7.76(1 \mathrm{H}, \mathrm{d}, J=16.0), 7.56-7.54(2 \mathrm{H}, \mathrm{m}), 7.39-7.36(3 \mathrm{H}$, $\mathrm{m}), 6.59(1 \mathrm{H}, \mathrm{d}, J=16.0), 5.12(1 \mathrm{H}, \mathrm{t}, J=7.0), 2.89-2.83(1 \mathrm{H}, \mathrm{m})$, 2.49-2.48 (1H, m), 2.06-2.03 (2H, m), $1.67(3 \mathrm{H}, \mathrm{s}), 1.60(3 \mathrm{H}, \mathrm{s})$, $1.26(3 \mathrm{H}, \mathrm{s}), 1.18$ (3H, s), $1.14(3 \mathrm{H}, \mathrm{s}), 1.06$ (3H, s), $0.99(3 \mathrm{H}, \mathrm{s})$, 0.87 (3H, s). ${ }^{13} \mathrm{C}-\mathrm{NMR}\left(125 \mathrm{MHz}, \mathrm{CDCl}_{3}, \delta_{\mathrm{C}}, \mathrm{ppm}\right): 175.74$, $165.25,147.71,134.52,131.61,130.38,128.90,128.15,124.71$, 116.43, 75.36, 55.95, 50.27, 49.87, 42.33, 41.60, 40.46, 40.40, $39.45,39.16,37.14,34.73,31.13,31.03,27.51,27.45,26.34$, $25.72,25.49,24.79,22.68,22.57,21.91,19.74,19.03,17.70$, 16.34, 16.17, 15.36 .

Compound $3 c \quad$ [20(S)-hydroxydammar-24-ene-3-oximylbenzoate]. White amorphous powder, $\mathrm{C}_{37} \mathrm{H}_{55} \mathrm{NO}_{3}$, HR-ESI-MS $(\mathrm{m} / \mathrm{z}): 584.4167[\mathrm{M}+\mathrm{Na}]^{+}$(calculated for $\mathrm{C}_{37} \mathrm{H}_{55} \mathrm{NO}_{3} \mathrm{Na}$ 584.4142), ESI-MS $(\mathrm{m} / \mathrm{z}): 562.2[\mathrm{M}+\mathrm{H}]^{+} ;{ }^{1} \mathrm{H}-\mathrm{NMR}(500 \mathrm{MHz}$, $\left.\mathrm{CDCl}_{3}, \delta_{\mathrm{H}}, \mathrm{ppm}\right),(J, \mathrm{~Hz}): 8.05(2 \mathrm{H}, \mathrm{d}, J=7.5), 7.56(1 \mathrm{H}, \mathrm{t}, J=7.5)$, $7.45(2 \mathrm{H}, \mathrm{t}, J=7.5), 5.12(1 \mathrm{H}, \mathrm{t}, J=7.5), 2.97-2.92(1 \mathrm{H}, \mathrm{m}), 2.58-$ $2.51(1 \mathrm{H}, \mathrm{m}), 2.06-2.03(2 \mathrm{H}, \mathrm{m}), 1.68(3 \mathrm{H}, \mathrm{s}), 1.62(3 \mathrm{H}, \mathrm{s}), 1.33$ (3H, s), 1.23 (3H, s), $1.14(3 \mathrm{H}, \mathrm{s}), 1.00(3 \mathrm{H}, \mathrm{s}), 0.97$ (3H, s), 0.83 (3H, s). ${ }^{13} \mathrm{C}-\mathrm{NMR}\left(125 \mathrm{MHz}, \mathrm{CDCl}_{3}, \delta_{\mathrm{C}}, \mathrm{ppm}\right):$ 176.48, 164.25, $132.94,131.59,129.79,129.50,128.44,124.71,75.35,55.95$, $50.26,49.87,42.33,41.70,40.45,40.40,39.47,37.16,34.73$, $31.58,31.14,27.50,27.47,25.72,25.49,24.79,22.72,22.57$, 21.92, 19.98, 19.03, 17.69, 16.34, 16.16, 15.36 .

Compound $\quad 3 d \quad\{20(S)$-hydroxydammar-24-ene-3-oximyl-[2hydroxy-3,5-diiodobenzoate]\}. White amorphous powder, $\mathrm{C}_{37} \mathrm{H}_{53} \mathrm{NO}_{4} \mathrm{I}_{2}$, HR-ESI-MS ( $\mathrm{m} / \mathrm{z}$ ): $852.1983[\mathrm{M}+\mathrm{Na}]^{+}$(calculated for $\mathrm{C}_{37} \mathrm{H}_{53} \mathrm{NO}_{4} \mathrm{I}_{2} \mathrm{Na}$ 852.1962), ESI-MS $(\mathrm{m} / \mathrm{z}): 828.0[\mathrm{M}-\mathrm{H}]^{-} ;{ }^{1} \mathrm{H}^{-}$ NMR (500 MHz, $\left.\mathrm{CDCl}_{3}, \delta_{\mathrm{H}}, \mathrm{ppm}\right),(J, \mathrm{~Hz}): 11.65(1 \mathrm{H}, \mathrm{s}, \mathrm{OH})$, $8.21(1 \mathrm{H}, \mathrm{d}, J=2.5), 8.07(1 \mathrm{H}, \mathrm{d}, J=2.5), 5.13-5.10(1 \mathrm{H}, \mathrm{m})$, 2.92-2.87 (1H, m), 2.60-2.54 (1H, m), 2.06-2.03 (2H, m), 1.75 $(3 \mathrm{H}, \mathrm{s}), 1.65(3 \mathrm{H}, \mathrm{s}), 1.29(3 \mathrm{H}, \mathrm{s}), 1.21(3 \mathrm{H}, \mathrm{s}), 1.14(3 \mathrm{H}, \mathrm{s}), 1.00$ $(3 \mathrm{H}, \mathrm{s}), 0.98(3 \mathrm{H}, \mathrm{s}), 0.88(3 \mathrm{H}, \mathrm{s}) .{ }^{13} \mathrm{C}-\mathrm{NMR}\left(125 \mathrm{MHz}, \mathrm{CDCl}_{3}, \delta_{\mathrm{C}}\right.$, ppm): 178.20, 166.54, 160.49, 152.17, 137.72, 131.65, 124.68, $113.61,87.23,80.62,75.35,55.93,50.26,49.87,42.32,42.02$, $40.43,40.40,39.44,37.17,34.68,31.13,27.47,27.44,25.72$, $25.53,24.79,22.72,22.57,21.94,20.34,19.00,17.70,16.34$, $16.17,15.36$.

Compound $3 \boldsymbol{e}\{20($ S)-hydroxydammar-24-ene-3-oximyl-[6-chloronicotinoate]\}. White amorphous powder, $\mathrm{C}_{36} \mathrm{H}_{53} \mathrm{ClN}_{2} \mathrm{O}_{3}, \mathrm{HR}-$ ESI-MS $(\mathrm{m} / \mathrm{z}): 619.3785[\mathrm{M}+\mathrm{Na}]^{+}$(calculated for $\mathrm{C}_{36} \mathrm{H}_{53} \mathrm{ClN}_{2}-$ $\mathrm{O}_{3} \mathrm{Na}$ 619.3759), ESI-MS $(\mathrm{m} / \mathrm{z}): 598.2[\mathrm{M}+\mathrm{H}]^{+} ;{ }^{1} \mathrm{H}-\mathrm{NMR}(500$ $\left.\mathrm{MHz}, \mathrm{CDCl}_{3}, \delta_{\mathrm{H}}, \mathrm{ppm}\right),(J, \mathrm{~Hz}): 9.00(1 \mathrm{H}, \mathrm{d}, J=2.0), 8.26(1 \mathrm{H}, \mathrm{dd}$, $J=8.0,2.0), 7.44(1 \mathrm{H}, \mathrm{d}, J=8.0), 5.12(1 \mathrm{H}, \mathrm{d}, J=7.0), 2.92-2.87$ (1H, m), 2.58-2.52 (1H, m), 2.06-2.04 (2H, m), $1.75(3 \mathrm{H}, \mathrm{s}), 1.66$ $(3 \mathrm{H}, \mathrm{s}), 1.33$ (3H, s), $1.23(3 \mathrm{H}, \mathrm{s}), 1.14(3 \mathrm{H}, \mathrm{s}), 1.00(3 \mathrm{H}, \mathrm{s}), 0.87$ (3H, s). ${ }^{13} \mathrm{C}-\mathrm{NMR}\left(125 \mathrm{MHz}, \mathrm{CDCl}_{3}, \delta_{\mathrm{C}}, \mathrm{ppm}\right): 177.35,162.14$, $155.70,150.79$, 139.55, 131.58, 124.79, 124.70, 124.37, 75.31,
$55.91,50.25,49.84,42.30,41.85,40.47,40.38,39.41,37.15$, $34.69,31.13,27.46,25.72,25.49,24.78,22.69,22.56,21.92$, $20.12,19.01,17.70,16.33,16.17,15.35$.

Compound $3 f\{20(S)$-hydroxydammar-24-ene-3-oximyl-[4-chlorobenzoate]\}. White amorphous powder, $\mathrm{C}_{37} \mathrm{H}_{54} \mathrm{ClNO}_{3}$, HR-ESIMS $(\mathrm{m} / \mathrm{z}): 630.3467[\mathrm{M}+\mathrm{Cl}]^{-}$(calculated for $\mathrm{C}_{37} \mathrm{H}_{54} \mathrm{Cl}_{2} \mathrm{NO}_{3}$ 630.3486); ${ }^{1} \mathrm{H}-\mathrm{NMR}\left(500 \mathrm{MHz}, \mathrm{CDCl}_{3}, \delta_{\mathrm{H}}, \mathrm{ppm}\right),(J, \mathrm{~Hz}): 7.98$ $(2 \mathrm{H}, \mathrm{d}, J=8.5), 7.43$ (2H, d, $J=8.5), 5.13-5.10$ (1H, m), 2.94-2.89 $(1 \mathrm{H}, \mathrm{m}), 2.57-2.50(1 \mathrm{H}, \mathrm{m}), 2.06-2.03(2 \mathrm{H}, \mathrm{m}), 1.67(3 \mathrm{H}, \mathrm{s}), 1.62$ $(3 \mathrm{H}, \mathrm{s}), 1.33(3 \mathrm{H}, \mathrm{s}), 1.24(3 \mathrm{H}, \mathrm{s}), 1.22(3 \mathrm{H}, \mathrm{s}), 1.08(3 \mathrm{H}, \mathrm{s}), 1.06$ $(3 \mathrm{H}, \mathrm{s}), 0.87(3 \mathrm{H}, \mathrm{s}) .{ }^{13} \mathrm{C}-\mathrm{NMR}\left(125 \mathrm{MHz}, \mathrm{CDCl}_{3}, \delta_{\mathrm{C}}, \mathrm{ppm}\right)$ : 176.73, 163.41, 139.43, 131.60, 130.86, 128.83, 128.23, 124.71, $75.34,55.94,50.26,49.89,42.33,41.75,40.45,40.41,39.45$, $37.17,34.73,31.13,27.48,25.71,25.51,24.79,22.72,22.57$, $21.93,20.02,19.02,17.69,16.34,16.16,15.36$.

Compound $3 \mathrm{~g}\{20($ S)-hydroxydammar-24-ene-3-oximyl-[4-fluorobenzoate]\}. White amorphous powder, $\mathrm{C}_{37} \mathrm{H}_{54} \mathrm{NO}_{3} \mathrm{~F}$, HR-ESIMS $(m / z): 602.4125[\mathrm{M}+\mathrm{Na}]^{+}$(calculated for $\mathrm{C}_{37} \mathrm{H}_{54} \mathrm{NO}_{3} \mathrm{FNa}$ 602.4097); ${ }^{1} \mathrm{H}-\mathrm{NMR}\left(500 \mathrm{MHz}, \mathrm{CDCl}_{3}, \delta_{\mathrm{H}}, \mathrm{ppm}\right),(J, \mathrm{~Hz}): 8.07$ $(2 \mathrm{H}, \mathrm{dd}, J=8.0,2.0), 7.12(2 \mathrm{H}, \mathrm{dd}, J=8.0,2.0), 5.12(1 \mathrm{H}, \mathrm{t}, J=$ 7.0), 2.94-2.91 (1H, m), 2.59-2.51 $(1 \mathrm{H}, \mathrm{m}), 2.06-2.03(2 \mathrm{H}, \mathrm{m})$, $1.75(3 \mathrm{H}, \mathrm{s}), 1.71(3 \mathrm{H}, \mathrm{s}), 1.32(3 \mathrm{H}, \mathrm{s}), 1.20(3 \mathrm{H}, \mathrm{s}), 1.14(3 \mathrm{H}, \mathrm{s})$, $1.00(3 \mathrm{H}, \mathrm{s}), 0.97(3 \mathrm{H}, \mathrm{s}), 0.87(3 \mathrm{H}, \mathrm{s}) .{ }^{13} \mathrm{C}-\mathrm{NMR}(125 \mathrm{MHz}$, $\left.\mathrm{CDCl}_{3}, \delta_{\mathrm{C}}, \mathrm{ppm}\right): 176.58,166.76,163.30,132.05,131.97,131.59$, $126.00,125.97,124.71,115.55,75.33,55.93,50.26,49.87,42.32$, $41.72,40.45,40.40,39.45,37.16,34.72,31.13,27.49,27.47$, $25.72,25.50,24.79,22.71,22.57,21.92,20.00,19.02,17.69$, 16.34, 16.16, 15.35 .

Compound $3 \boldsymbol{h}\{20(S)$-hydroxydammar-24-ene-3-oximyl-[2-iodobenzoate]\}. White amorphous powder, $\mathrm{C}_{37} \mathrm{H}_{54} \mathrm{NO}_{3} \mathrm{I}$, HR-ESI-MS $(\mathrm{m} / \mathrm{z}): 710.3213[\mathrm{M}+\mathrm{Na}]^{+}$(calculated for $\mathrm{C}_{37} \mathrm{H}_{54} \mathrm{NO}_{3} \mathrm{INa}$ 710.3170); ${ }^{1} \mathrm{H}-\mathrm{NMR}\left(500 \mathrm{MHz}, \mathrm{CDCl}_{3}, \delta_{\mathrm{H}}, \mathrm{ppm}\right),(J, \mathrm{~Hz}): 7.97$ $(1 \mathrm{H}, \mathrm{dd}, J=8.0,1.0), 7.73(1 \mathrm{H}, \mathrm{dd}, J=8.0,2.0), 7.42-7.39(1 \mathrm{H}$, $\mathrm{m}), 7.17-7.13(1 \mathrm{H}, \mathrm{m}), 5.13-5.10(1 \mathrm{H}, \mathrm{m}), 2.96-2.92(1 \mathrm{H}, \mathrm{m})$, 2.58-2.52 (1H, m), 2.06-2.03 (2H, m), $1.68(3 \mathrm{H}, \mathrm{s}), 1.62(3 \mathrm{H}, \mathrm{s})$, $1.30(3 \mathrm{H}, \mathrm{s}), 1.22(3 \mathrm{H}, \mathrm{s}), 1.19(3 \mathrm{H}, \mathrm{s}), 0.99(3 \mathrm{H}, \mathrm{s}), 0.96(3 \mathrm{H}, \mathrm{s})$, $0.83(3 \mathrm{H}, \mathrm{s}) .{ }^{13} \mathrm{C}-\mathrm{NMR}\left(125 \mathrm{MHz}, \mathrm{CDCl}_{3}, \delta_{\mathrm{C}}, \mathrm{ppm}\right): 176.66$, $164.81,140.96,135.84,132.35,131.54,130.66,127.84,124.70$, $93.60,75.30,55.96,50.26,50.25,49.86,42.31,41.75,40.45$, $40.39,39.56,37.15,34.72,31.12,27.48,27.41,25.69,25.49$, $24.78,22.62,22.55,21.91,20.23,19.00,17.67,16.34,16.21$, 15.34 .

Compound $3 \boldsymbol{i}\{20($ S)-hydroxydammar-24-ene-3-oximyl-[3-fluorobenzoate]\}. White amorphous powder, $\mathrm{C}_{37} \mathrm{H}_{54} \mathrm{FNO}_{3}$, HRESIMS (m/z): $602.3981[\mathrm{M}+\mathrm{Na}]^{+}$(calculated for $\mathrm{C}_{37} \mathrm{H}_{54} \mathrm{FNO}_{3} \mathrm{Na}$ 602.3985); ${ }^{1} \mathrm{H}-\mathrm{NMR}\left(500 \mathrm{MHz}, \mathrm{CDCl}_{3}, \delta_{\mathrm{H}}, \mathrm{ppm}\right),(J, \mathrm{~Hz}): 7.85$ $(1 \mathrm{H}, \mathrm{dd}, J=7.5,1.0), 7.72(1 \mathrm{H}, \mathrm{dd}, J=7.5,4.0), 7.45-7.41(1 \mathrm{H}$, $\mathrm{m}), 7.29-7.25(1 \mathrm{H}, \mathrm{m}), 5.13-5.10(1 \mathrm{H}, \mathrm{m}), 2.95-2.90(1 \mathrm{H}, \mathrm{m})$, 2.58-2.52 (1H, m), 2.06-2.03 (2H, m), $1.68(3 \mathrm{H}, \mathrm{s}), 1.62(3 \mathrm{H}, \mathrm{s})$, $1.32(3 \mathrm{H}, \mathrm{s}), 1.21(3 \mathrm{H}, \mathrm{s}), 1.14(3 \mathrm{H}, \mathrm{s}), 1.10(3 \mathrm{H}, \mathrm{s}), 0.97(3 \mathrm{H}, \mathrm{s})$, 0.87 (3H, s). ${ }^{13} \mathrm{C}-\mathrm{NMR}\left(125 \mathrm{MHz}, \mathrm{CDCl}_{3}, \delta_{\mathrm{C}}, \mathrm{ppm}\right): 176.75$, $163.51,161.54,131.93,131.87,131.50,130.11,130.05,125.20$, 124.69, 75.26, 55.89, 50.22, 49.83, 42.28, 41.70, 40.43, 40.36, $39.40,37.12,34.68,31.09,27.45,25.67,25.45,24.75,22.67$, 22.53, 21.89, 19.98, 18.98, 17.64, 16.30, 16.11, 15.31 .

Compound 3k [20(S)-hydroxydammar-24-ene-3-oximyl acetate]. White amorphous powder, $\mathrm{C}_{32} \mathrm{H}_{53} \mathrm{NO}_{3}$, HR-ESI-MS $(\mathrm{m} / \mathrm{z})$ : 
$522.4045[\mathrm{M}+\mathrm{Na}]^{+}$(calculated for $\left.\mathrm{C}_{32} \mathrm{H}_{53} \mathrm{NO}_{3} \mathrm{Na} 522.3997\right) ;{ }^{1} \mathrm{H}-$ NMR (500 MHz, $\left.\mathrm{CDCl}_{3}, \delta_{\mathrm{H}}, \mathrm{ppm}\right),(J, \mathrm{~Hz}): 5.13-5.10(1 \mathrm{H}, \mathrm{m})$, 2.86-2.81 (1H, m), 2.45-2.39 (1H, m), 2.17 (3H, s, $\left.\mathrm{CH}_{3} \mathrm{COO}\right)$, 2.06-2.03 (2H, m), 1.67 (3H, s), 1.57 (3H, s), 1.23 (3H, s), 1.14 $(3 \mathrm{H}, \mathrm{s}), 1.13(3 \mathrm{H}, \mathrm{s}), 0.98(3 \mathrm{H}, \mathrm{s}), 0.94(3 \mathrm{H}, \mathrm{s}), 0.86(3 \mathrm{H}, \mathrm{s}) \cdot{ }^{13} \mathrm{C}-$ NMR (125 MHz, $\left.\mathrm{CDCl}_{3}, \delta_{\mathrm{C}}, \mathrm{ppm}\right): 174.86,169.85,131.57$, 124.70, 75.34, 55.89, 50.25, 49.84, 42.31, 41.39, 40.47, 40.38, $39.35,37.09,34.71,31.12,27.49,27.40,25.71,25.47,24.78$, $22.62,22.56,21.89,20.00,19.52,19.00,17.69,16.32,16.13$, 15.34 .

Compound 31 [20(S)-hydroxydammar-24-ene-3-oximyl pentanoate]. White amorphous powder, $\mathrm{C}_{35} \mathrm{H}_{59} \mathrm{NO}_{3}$, HR-ESI-MS $(\mathrm{m} / \mathrm{z})$ : $542.4588[\mathrm{M}+\mathrm{H}]^{+}$(calculated for $\left.\mathrm{C}_{35} \mathrm{H}_{60} \mathrm{NO}_{3} 542.4567\right) ;{ }^{1} \mathrm{H}-\mathrm{NMR}$ (500 MHz, $\left.\mathrm{CDCl}_{3}, \delta_{\mathrm{H}}, \mathrm{ppm}\right),(J, \mathrm{~Hz}): 5.13-5.10(1 \mathrm{H}, \mathrm{m}), 2.84-2.79$ $(1 \mathrm{H}, \mathrm{m}), 2.42\left(2 \mathrm{H}, \mathrm{t}, J=7.5, \mathrm{OCOCH}_{2}\right), 2.41-2.37(1 \mathrm{H}, \mathrm{m}), 2.07-$ $2.01(2 \mathrm{H}, \mathrm{m}), 1.70(3 \mathrm{H}, \mathrm{s}), 1.62(3 \mathrm{H}, \mathrm{s}), 1.24(3 \mathrm{H}, \mathrm{s}), 1.15(3 \mathrm{H}, \mathrm{s})$, $1.14(3 \mathrm{H}, \mathrm{s}), 0.98(3 \mathrm{H}, \mathrm{s}), 0.94(3 \mathrm{H}, \mathrm{s}), 0.92(3 \mathrm{H}, \mathrm{t}, J=7.5), 0.86$ (3H, s). ${ }^{13} \mathrm{C}-\mathrm{NMR}\left(125 \mathrm{MHz}, \mathrm{CDCl}_{3}, \delta_{\mathrm{C}}, \mathrm{ppm}\right):$ 175.07, 172.07, 131.56, 124.71, 75.33, 55.90, 50.25, 49.85, 42.32, 41.45, 40.46, $40.38,39.37,37.10,34.72,32.92,31.12,27.49,25.47,24.78$, $22.63,22.56,22.31,21.89,19.55,19.00,17.68,16.32,16.11$, 15.34, 13.69.

Compound $3 \mathrm{~m}$ [20(S)-hydroxydammar-24-ene-3-oximyl isobutyrate]. White amorphous powder, $\mathrm{C}_{34} \mathrm{H}_{57} \mathrm{NO}_{3}$, HR-ESI-MS $(m / z): 550.4378[\mathrm{M}+\mathrm{Na}]^{+}$(calculated for $\mathrm{C}_{34} \mathrm{H}_{57} \mathrm{NO}_{3} \mathrm{Na}$ 550.4330), ESI-MS $(\mathrm{m} / \mathrm{z}): 528.2[\mathrm{M}+\mathrm{H}]^{+} ;{ }^{1} \mathrm{H}-\mathrm{NMR}(500 \mathrm{MHz}$, $\left.\mathrm{CDCl}_{3}, \delta_{\mathrm{H}}, \mathrm{ppm}\right),(\mathrm{J}, \mathrm{Hz}): 5.13-5.10(1 \mathrm{H}, \mathrm{m}), 2.85-2.79(1 \mathrm{H}, \mathrm{m})$, 2.72-2.66 (1H, m), 2.43-2.37 (1H, m), 2.07-2.03 (2H, m), 1.69 $(3 \mathrm{H}, \mathrm{s}), 1.62(3 \mathrm{H}, \mathrm{s}), 1.25(3 \mathrm{H}, \mathrm{s}), 1.23(6 \mathrm{H}, \mathrm{d}, J=7.0), 1.14(6 \mathrm{H}$, brs), 0.98 (3H, s), 0.94 (3H, s), 0.86 (3H, s). ${ }^{13} \mathrm{C}-\mathrm{NMR}(125 \mathrm{MHz}$, $\left.\mathrm{CDCl}_{3}, \delta_{\mathrm{C}}, \mathrm{ppm}\right): 175.59,174.79,131.61,124.70,75.39,55.93$, $50.25,49.86,42.32,41.53,40.44,40.39,39.39,37.11,34.73$, $33.19,31.12,27.49,27.33,25.71,25.48,24.79,22.65,22.56$, 21.89, 19.54, 19.06, 19.03, 18.98, 17.69, 16.32, 16.10, 15.35.

Compound 4 \{17-[(5 $\alpha$-methyl)-3,4H-furane-2-one]-25,26,27-trisnor-dammar-3-one\}. White needles, $\mathrm{mp}$ 118.4-120.3 ${ }^{\circ} \mathrm{C}$; $\mathrm{C}_{27} \mathrm{H}_{42} \mathrm{O}_{3}$, HR-ESI-MS $(\mathrm{m} / \mathrm{z})$ : $415.3226[\mathrm{M}+\mathrm{H}]^{+}$(calculated for $\mathrm{C}_{27} \mathrm{H}_{43} \mathrm{O}_{3}$ 415.3206), ESI-MS $(\mathrm{m} / \mathrm{z}): 415.1[\mathrm{M}+\mathrm{H}]^{+}, 397.1\left[\mathrm{M}-\mathrm{H}_{2} \mathrm{O}\right.$ $+\mathrm{H}]^{+} ;{ }^{1} \mathrm{H}-\mathrm{NMR}\left(500 \mathrm{MHz}, \mathrm{CDCl}_{3}, \delta_{\mathrm{H}}, \mathrm{ppm}\right),(J, \mathrm{~Hz}): 2.55-2.50$ (1H, m, H-23a), 2.49-2.47 (1H, m, H-22a), 2.45-2.41 (1H, m, H23b), 2.38-2.35 (1H, m, H-2a), 2.06-2.04 (1H, m, H-17), 1.30 (3H, s, H-21), 1.01 (3H, s, H-25), 0.97 (3H, s, H-26), 0.93 (3H, s, H18), 0.87 (3H, s, H-19), 0.83 (3H, s, H-27). ${ }^{13} \mathrm{C}-\mathrm{NMR}(125 \mathrm{MHz}$,

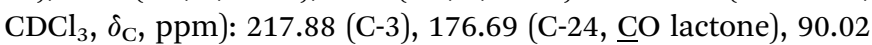
(C-20), 55.34 (C-5), 50.15, 49.93 (C-9), 49.30 (C-17), 47.40, 43.31 (C-13), 40.28, 39.85 36.83, 34.53, 34.07, 31.18, 31.09, 29.16, $26.83,26.70,25.49,25.02,21.93,21.02$, 19.61, 16.16, 15.98, 15.19 .

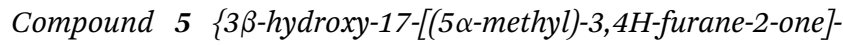
25,26,27-tris-nor-dammarane\}. White needles, $\mathrm{mp}$ 195.0196.0 ${ }^{\circ} \mathrm{C}, \mathrm{C}_{27} \mathrm{H}_{44} \mathrm{O}_{3}$, HR-ESI-MS $(\mathrm{m} / \mathrm{z}): 417.3378[\mathrm{M}+\mathrm{H}]^{+}$ (calculated for $\mathrm{C}_{27} \mathrm{H}_{45} \mathrm{O}_{3}$ 417.3363); ${ }^{1} \mathrm{H}$-NMR (500 MHz, $\mathrm{CDCl}_{3}$, $\left.\delta_{\mathrm{H}}, \mathrm{ppm}\right),(J, \mathrm{~Hz}): 3.19(1 \mathrm{H}, \mathrm{dd}, J=11.5,5.0), 2.68-2.62(1 \mathrm{H}, \mathrm{m})$, 2.57-2.50 (1H, m), 2.15-2.08 (1H, m), 2.01-1.90 (2H, m), 1.37 $(3 \mathrm{H}, \mathrm{s}), 0.98(3 \mathrm{H}, \mathrm{s}), 0.97(3 \mathrm{H}, \mathrm{s}), 0.88(3 \mathrm{H}, \mathrm{s}), 0.85(3 \mathrm{H}, \mathrm{s}), 0.77$
(3H, s). ${ }^{13} \mathrm{C}-\mathrm{NMR}\left(125 \mathrm{MHz}, \mathrm{CDCl}_{3}, \delta_{\mathrm{C}}, \mathrm{ppm}\right):$ 176.75, 90.14, 78.93, 55.85, 50.58, 50.18, 49.37, 43.20, 40.38, 39.04, 38.98, $37.14,35.21$, 31.21, 31.10, 29.20, 28.00, 27.40, 26.82, 25.44, $25.05,21.43,18.25,16.25,16.18,15.48,15.35$.

Compound $\quad 6 \boldsymbol{a} \quad\{17-[(5 \alpha-m e t h y l)-3,4 H$-furane-2-one $]-3 \beta-(O-$ acetyl)-25,26,27-tris-nor-dammarane\}. White needles, mp 248.0$249.0{ }^{\circ} \mathrm{C}, \mathrm{C}_{29} \mathrm{H}_{46} \mathrm{O}_{4}$, HR-ESI-MS $(\mathrm{m} / \mathrm{z}): 481.3357[\mathrm{M}+\mathrm{Na}]^{+}$ (calculated for $\left.\mathrm{C}_{29} \mathrm{H}_{46} \mathrm{O}_{4} \mathrm{Na} 481.3294\right)$, ESI-MS $(\mathrm{m} / \mathrm{z}): 459.1[\mathrm{M}+$ $\mathrm{H}]^{+} ;{ }^{1} \mathrm{H}-\mathrm{NMR}\left(500 \mathrm{MHz}, \mathrm{CDCl}_{3}, \delta_{\mathrm{H}}, \mathrm{ppm}\right),(J, \mathrm{~Hz}): 4.48(1 \mathrm{H}, \mathrm{dd}, J$ $=11.0,5.5), 2.68-2.62(1 \mathrm{H}, \mathrm{m}), 2.57-2.50(1 \mathrm{H}, \mathrm{m}), 2.14-2.10(1 \mathrm{H}$, $\mathrm{m}), 2.04\left(3 \mathrm{H}, \mathrm{s}, \mathrm{CH}_{3} \mathrm{COO}\right), 2.00-1.90(2 \mathrm{H}, \mathrm{m}), 1.37(3 \mathrm{H}, \mathrm{s})$, 0.96 (3H, s), 0.88 (3H, s), 0.86 (3H, s), 0.84 (6H, brs). ${ }^{13} \mathrm{C}-\mathrm{NMR}$ (125 MHz, $\left.\mathrm{CDCl}_{3}, \delta_{\mathrm{C}}, \mathrm{ppm}\right): 176.75,170.98,90.10,80.86$, 55.93 , 50.50, 50.16, 49.36, 43.18, 40.39, 38.71, 37.90, 37.05, $35.14,31.22$, 31.19, 29.18, 27.96, 26.80, 25.37, 25.04, 23.68, $21.43,21.29,18.13,16.48,16.24,16.22,15.48$.

Compound $\mathbf{6 b}\{17-[(5 \alpha-$ methyl)-3,4H-furane-2-one $]-3 \beta-(O-p e n-$ tanoyl)-25,26,27-tris-nor-dammarane $\}$. White needles, mp 134.0135.0 ${ }^{\circ} \mathrm{C}, \mathrm{C}_{32} \mathrm{H}_{52} \mathrm{O}_{4}$, HR-ESI-MS $(\mathrm{m} / \mathrm{z}): 523.3892[\mathrm{M}+\mathrm{Na}]^{+}$ (calculated for $\left.\mathrm{C}_{32} \mathrm{H}_{52} \mathrm{O}_{4} \mathrm{Na} 523.3858\right)$, ESI-MS $(\mathrm{m} / \mathrm{z}): 501.3[\mathrm{M}+$ $\mathrm{H}]^{+} ;{ }^{1} \mathrm{H}-\mathrm{NMR}\left(500 \mathrm{MHz}, \mathrm{CDCl}_{3}, \delta_{\mathrm{H}}, \mathrm{ppm}\right),(J, \mathrm{~Hz}): 4.48(1 \mathrm{H}, \mathrm{dd}, J$ $=11.0,5.0), 2.65-2.60(1 \mathrm{H}, \mathrm{m}), 2.56-2.50(1 \mathrm{H}, \mathrm{m}), 2.29(2 \mathrm{H}, \mathrm{t}, J=$ 7.5), 2.14-2.10 (1H, m), 2.01-1.90 (2H, m), $1.37(3 \mathrm{H}, \mathrm{s}), 0.96(3 \mathrm{H}$, s), $0.91(3 \mathrm{H}, \mathrm{t}, J=7.5), 0.88(3 \mathrm{H}, \mathrm{s}), 0.86(3 \mathrm{H}, \mathrm{s}), 0.85$ (6H, brs). ${ }^{13} \mathrm{C}-\mathrm{NMR}\left(125 \mathrm{MHz}, \mathrm{CDCl}_{3}, \delta_{\mathrm{C}}, \mathrm{ppm}\right): 176.73,173.64,90.09$, $80.50,55.93,50.48,50.17,49.37,43.19,40.40,38.70,37.94$, $37.06,35.14,34.56,31.22,31.19,29.17,27.97,27.23,26.80$, $25.36,25.04,23.72,22.29,21.44,18.13,16.54,16.23,15.48$, 13.70 .

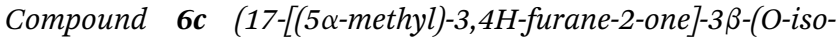
butyryl)-25,26,27-tris-nor-dammarane). White needle crystals, mp 206.0-207.0 ${ }^{\circ} \mathrm{C} ; \mathrm{C}_{31} \mathrm{H}_{50} \mathrm{O}_{4}$, HR-ESI-MS $(\mathrm{m} / \mathrm{z}): 509.3683[\mathrm{M}+$ $\mathrm{Na}]^{+}$(calculated for $\mathrm{C}_{31} \mathrm{H}_{50} \mathrm{O}_{4} \mathrm{Na} 509.3671$ ), ESI-MS $(\mathrm{m} / \mathrm{z}): 487.2$ $[\mathrm{M}+\mathrm{H}]^{+} ;{ }^{1} \mathrm{H}-\mathrm{NMR}\left(500 \mathrm{MHz}, \mathrm{CDCl}_{3}, \delta_{\mathrm{H}}, \mathrm{ppm}\right),(J, \mathrm{~Hz}): 4.48-4.44$ $(1 \mathrm{H}, \mathrm{m}), 2.68-2.62(1 \mathrm{H}, \mathrm{m}), 2.57-2.50(1 \mathrm{H}, \mathrm{m}), 2.14-2.10(1 \mathrm{H}$, $\mathrm{m}), 2.01-1.90(1 \mathrm{H}, \mathrm{m}), 1.39(3 \mathrm{H}, \mathrm{s}), 1.17(3 \mathrm{H}, \mathrm{d}, J=7.5), 1.16(3 \mathrm{H}$, $\mathrm{d}, J=7.5), 0.96(3 \mathrm{H}, \mathrm{s}), 0.88(3 \mathrm{H}, \mathrm{s}), 0.87(3 \mathrm{H}, \mathrm{s}), 0.86(3 \mathrm{H}, \mathrm{s})$, $0.84(3 \mathrm{H}, \mathrm{s}) .{ }^{13} \mathrm{C}-\mathrm{NMR}\left(125 \mathrm{MHz}, \mathrm{CDCl}_{3}, \delta_{\mathrm{C}}, \mathrm{ppm}\right): 176.79$, 176.73 , 90.09, 80.27, 55.93, 50.48, 50.18, 49.37, 43.19, 40.40, $38.67,38.06,37.07,35.14,34.47,31.23,31.20,29.17,27.95$, $26.80,25.36,25.04,23.64,21.44,19.18,18.93,18.11,16.54$, $16.25,16.22,15.48$.

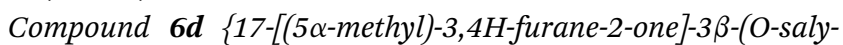
cyloyl)-25,26,27-tris-nor-dammarane $\}$. White needles, mp 186.0$187.0{ }^{\circ} \mathrm{C} ; \mathrm{C}_{34} \mathrm{H}_{48} \mathrm{O}_{5}$, HR-ESI-MS $(\mathrm{m} / \mathrm{z}): 559.3519[\mathrm{M}+\mathrm{Na}]^{+}$ (calculated for $\left.\mathrm{C}_{34} \mathrm{H}_{48} \mathrm{O}_{5} \mathrm{Na} 559.3494\right)$, ESI-MS $(\mathrm{m} / \mathrm{z}): 537.1[\mathrm{M}+$ $\mathrm{H}]^{+} ;{ }^{1} \mathrm{H}-\mathrm{NMR}\left(500 \mathrm{MHz}, \mathrm{CDCl}_{3}, \delta_{\mathrm{H}}, \mathrm{ppm}\right),(J, \mathrm{~Hz}): 7.83(1 \mathrm{H}, \mathrm{dd}, J$ $=8.0,2.0), 7.46-7.42(1 \mathrm{H}, \mathrm{dt}, J=7.5,1.5), 6.98(1 \mathrm{H}, \mathrm{dd}, J=8.0$, 1.0), 6.87 (dt, $J=8.0,1.0), 5.29(1 \mathrm{H}, \mathrm{s}, \mathrm{OH}), 4.77-4.74(1 \mathrm{H}, \mathrm{m})$, 2.67-2.61 (1H, m), 2.57-2.54 (1H, m), 2.13-2.09 (1H, m), 1.36 $(3 \mathrm{H}, \mathrm{s}), 1.01$ (3H, s), $0.98(3 \mathrm{H}, \mathrm{s}), 0.93(3 \mathrm{H}, \mathrm{s}), 0.92(3 \mathrm{H}, \mathrm{s}), 0.90$ $(3 \mathrm{H}, \mathrm{s}) .{ }^{13} \mathrm{C}-\mathrm{NMR}\left(125 \mathrm{MHz}, \mathrm{CDCl}_{3}, \delta_{\mathrm{C}}, \mathrm{ppm}\right):$ 176.70, 169.90, $161.74,135.44,129.76,119.04,117.58,113.09$, 90.06, 82.27, $56.00,50.51,50.19,49.37,43.19,40.43,38.70,38.30,37.11$, 
$35.13,31.20,29.69,29.17,28.14,26.81,25.41,25.04,23.71$, $21.48,18.15,16.73,16.25,15.51$.

Compound $6 \boldsymbol{e}$ \{17-[(5 $\alpha$-methyl)-3,4H-furane-2-one $]-3 \beta-(O-c i n n a-$ moyl)-25,26,27-tris-nor-dammarane $\}$. White crystals, mp 236.0238.0 ${ }^{\circ} \mathrm{C} ; \mathrm{C}_{36} \mathrm{H}_{50} \mathrm{O}_{4}$, HR-ESI-MS $(\mathrm{m} / \mathrm{z}): 583.5987\left[\mathrm{M}+\mathrm{H}+2 \mathrm{H}_{2} \mathrm{O}\right]^{+}$ (calculated for $\left.\mathrm{C}_{36} \mathrm{H}_{55} \mathrm{O}_{6} 583.3999\right),{ }^{1} \mathrm{H}-\mathrm{NMR}$ (500 $\mathrm{MHz}, \mathrm{CDCl}_{3}$, $\left.\delta_{\mathrm{H}}, \mathrm{ppm}\right),(J, \mathrm{~Hz}): 7.65(1 \mathrm{H}, \mathrm{d}, J=16.0), 7.53-7.51(2 \mathrm{H}, \mathrm{m}), 7.39-$ 7.37 (3H, m), 6.43 (1H, d, $J=16.0), 4.62(1 \mathrm{H}, \mathrm{dd}, J=11.0,5.0)$, 2.64-2.61 (1H, m), 2.57-2.54 (1H, m), 2.13-2.00 (1H, m), 1.50 $(3 \mathrm{H}, \mathrm{s}), 1.36$ (3H, s), 0.97 (3H, s), $0.93(3 \mathrm{H}, \mathrm{s}), 0.90$ (6H, brs). ${ }^{13} \mathrm{C}-$ NMR (125 MHz, $\left.\mathrm{CDCl}_{3}, \delta_{\mathrm{C}}, \mathrm{ppm}\right): 176.73,166.81,144.29,134.59$, 130.12, 128.85, 128.03, 118.88, 90.09, 80.94, 55.98, 50.52, 50.19, $49.38,43.21,40.42,38.76,38.17,37.10,35.16,31.25,31.20$, $29.18,28.04,26.82,25.36,25.05,23.80,21.46,18.16,16.65$, $16.25,15.50$.

3.2.2. Structural elucidation. Compound 1 was isolated from Dipterocarpus alatus as colourless needles. The NMR spectral data of this compound is consistent with the reported data. ${ }^{27,30}$ This compound was used as the starting material for the synthesis of the oxime and lactone derivatives.

Instead of a ketone signal $\left(\delta_{\mathrm{C}} 218.08\right)$ in the ${ }^{13} \mathrm{C}$ NMR spectrum of 1 , a signal at $\delta_{\mathrm{C}} 167.40$, which is characteristic of an oxime carbon at $\mathrm{C}-3$, was observed for compound 2 . The formation of oxime esters $\mathbf{3 a - 3 m}$ was confirmed by the appearance of carbonyl ester groups in the range of $\delta_{\mathrm{C}} 178.02-$ 174.48 in their ${ }^{13} \mathrm{C}$ NMR spectra. The signals representing the aromatic carbons of compounds $\mathbf{3 a}-\mathbf{3 i}$ are resonated at $\delta_{\mathrm{C}}$ 163.03-124.37 and $\delta_{\mathrm{H}}$ 9.17-7.12 in their NMR spectra. The ${ }^{1} \mathrm{H}$ NMR spectrum of compound 3k provides an additional methyl signal at $\delta_{\mathrm{H}} 2.17\left(3 \mathrm{H}, \mathrm{s}, \mathrm{CH}_{3} \mathrm{COO}\right)$ The signals at $\delta_{\mathrm{H}} 2.42$ $\left(2 \mathrm{H}, \mathrm{t}, J=7.5,-\mathrm{OCOCH}_{2}-\right)$, and 0.92 $\left(3 \mathrm{H}, \mathrm{t}, J=7.5,-\mathrm{CH}_{2} \underline{\mathrm{CH}_{3}}\right)$ demonstrate the presence of a pentanoyl group in compound 31. The presence of an isobutyryl group is inferred by the signal at $\delta_{\mathrm{H}} 1.23(6 \mathrm{H}, \mathrm{d}, J=6.0)$ in the ${ }^{1} \mathrm{H}$ NMR spectrum of compound $\mathbf{3 m}$. The NMR spectra of compound 4 indicate the disappearance of the olefine bond at C-24 and C-25 and the two methyl groups at C-26, C-27, compared with compound 1 . The presence of a carbonyl lactone at C-24 $\left(\delta_{\mathrm{C}} 176.69\right)$, together with the much more downfield chemical shift of the tertiary oxygenated carbon $\left(\delta_{\mathrm{C}} 90.02\right)$ suggests the formation of a lactone ring at the side chain of the dipterocarpol skeleton in compound $\mathbf{4}$. The structure of 4 was further confirmed by 2D NMR (COSY, HSQC, HMBC, and NOESY) spectra. The HMBC correlations between $\mathrm{H}-23\left(\delta_{\mathrm{H}} 2.55-\right.$ $2.50), \mathrm{H}-22\left(\delta_{\mathrm{H}} 2.49-2.47\right) / \mathrm{C}-24\left(\delta_{\mathrm{C}} 176.69\right), \mathrm{C}-20\left(\delta_{\mathrm{C}} 90.02\right), \mathrm{H}-21$ $\left(\delta_{\mathrm{H}} 1.30\right) / \mathrm{C}-20\left(\delta_{\mathrm{C}} 90.02\right)$ and C-17 (49.30) confirm the location of the lactone ring at $\mathrm{C}-17$. The orientation of the methyl group (C21) was established as $\alpha$ by the NOESY correlation between $\mathrm{H}-17$ $\left(\delta_{\mathrm{H}} 2.06-2.04\right)$ and $\mathrm{H}-21\left(\delta_{\mathrm{H}} 1.30\right)$.

The ${ }^{1} \mathrm{H}$ NMR spectrum of compound 5 presents a hydroxymethine group at $\delta_{\mathrm{H}} 3.19(1 \mathrm{H}, \mathrm{dd}, J=11.5,5.0, \mathrm{H}-3)$. The large coupling constant $(J=11.5)$ suggests the axial orientation of proton $\mathrm{H}-3$ and the $\beta$-configuration of the hydroxyl group at C-3.
An oxygenated methine carbon $\left(\delta_{\mathrm{C}} 78.93\right)$ was observed in the ${ }^{13} \mathrm{C}$ NMR spectrum of compound 5 , instead of a ketone group $\left(\delta_{\mathrm{C}} 217.88\right)$ in compound 4 . The ${ }^{13} \mathrm{C}$ NMR spectra of compounds 6a-6e contain additional signals of carbonyl ester in the range of $\delta_{\mathrm{C}} 176.63-170.98$. The signals at $\delta_{\mathrm{C}} 144.29-113.09$ and $\delta_{\mathrm{H}}$ 7.83-6.43 indicate the presence of aromatic rings in compounds 6d and 6e. A methyl carbonyl ester at $\delta_{\mathrm{H}} 2.04$ $\left(3 \mathrm{H}, \mathrm{s}, \mathrm{CH}_{3} \mathrm{COO}\right), \quad$ a pentanoyl group at $\delta_{\mathrm{H}} \quad 2.29$ $\left(2 \mathrm{H}, \mathrm{t}, J=7.5,-\mathrm{OCOC}^{2}-\right)$, and an isobutyryl group at $\delta_{\mathrm{H}} 1.17(3 \mathrm{H}, \mathrm{d}, J=7.5), 1.16(3 \mathrm{H}, \mathrm{d}, J=7.5)$ were determined from the ${ }^{1} \mathrm{H}$ NMR spectra of compounds $6 \mathbf{a}, \mathbf{6 b}$ and $6 \mathbf{c}$, respectively.

To the best of our knowledge, the twelve oxime esters and seven lactones are new dammarane derivatives that have not been reported in the literature to date. The analysis of the HRESIMS spectra data of all these newly synthesised compounds provided in Section 3.2 (characteristics) also further confirms the elucidation of their structures.

\subsection{Enzyme inhibition}

3.3.1. Experimental assays of $\alpha$-glucosidase inhibition. Twenty-one compounds $(\mathbf{1}, \mathbf{2}, \mathbf{3 a - 3 m}, \mathbf{4}, \mathbf{5}$, and 6a-6e) were evaluated for their ability to inhibit $\alpha$-glucosidase in vitro, and the corresponding results are given in Table 1.

The starting material dipterocarpol (1) was also tested for comparison with its synthetic derivatives. Acarbose was used as the standard compound in this assay. The results showed that dipterocarpol possesses weak activity ( $\left.\mathrm{IC}_{50} 264.91 \mu \mathrm{M}\right)$ compared with acarbose $\left(\mathrm{IC}_{50} 183.44 \mu \mathrm{M}\right)$. Nevertheless, most of

Table 1 Assay-based inhibition of the investigated compounds (dipterocarpol and its derivatives) towards $\alpha$-glucosidase ${ }^{a}$

\begin{tabular}{|c|c|c|}
\hline Number & Sample/compound & $\mathrm{IC}_{50}$ value $\pm \mathrm{SD}(\mu \mathrm{M})$ \\
\hline 1 & 1 & $264.91 \pm 9.64$ \\
\hline 2 & 2 & $36.94 \pm 2.80$ \\
\hline 3 & $3 \mathbf{a}$ & NA \\
\hline 4 & $3 \mathbf{b}$ & $33.12 \pm 5.37$ \\
\hline 5 & $3 c$ & $16.38 \pm 1.00$ \\
\hline 6 & 3d & NA \\
\hline 7 & $3 e$ & $36.60 \pm 1.26$ \\
\hline 8 & $3 f$ & $23.98 \pm 1.29$ \\
\hline 9 & $3 g$ & NA \\
\hline 10 & $3 h$ & NA \\
\hline 11 & $3 \mathbf{i}$ & $46.79 \pm 5.33$ \\
\hline 12 & $3 k$ & $59.15 \pm 2.08$ \\
\hline 13 & 31 & $43.51 \pm 1.68$ \\
\hline 14 & $3 m$ & NA \\
\hline 15 & 4 & NA \\
\hline 16 & 5 & $2.73 \pm 0.05$ \\
\hline 17 & $6 a$ & $220.5 \pm 4.77$ \\
\hline 18 & $6 b$ & $31.95 \pm 0.69$ \\
\hline 19 & $6 c$ & $4.62 \pm 0.12$ \\
\hline 20 & 6d & $228.09 \pm 4.62$ \\
\hline 21 & $6 e$ & $7.31 \pm 0.11$ \\
\hline
\end{tabular}



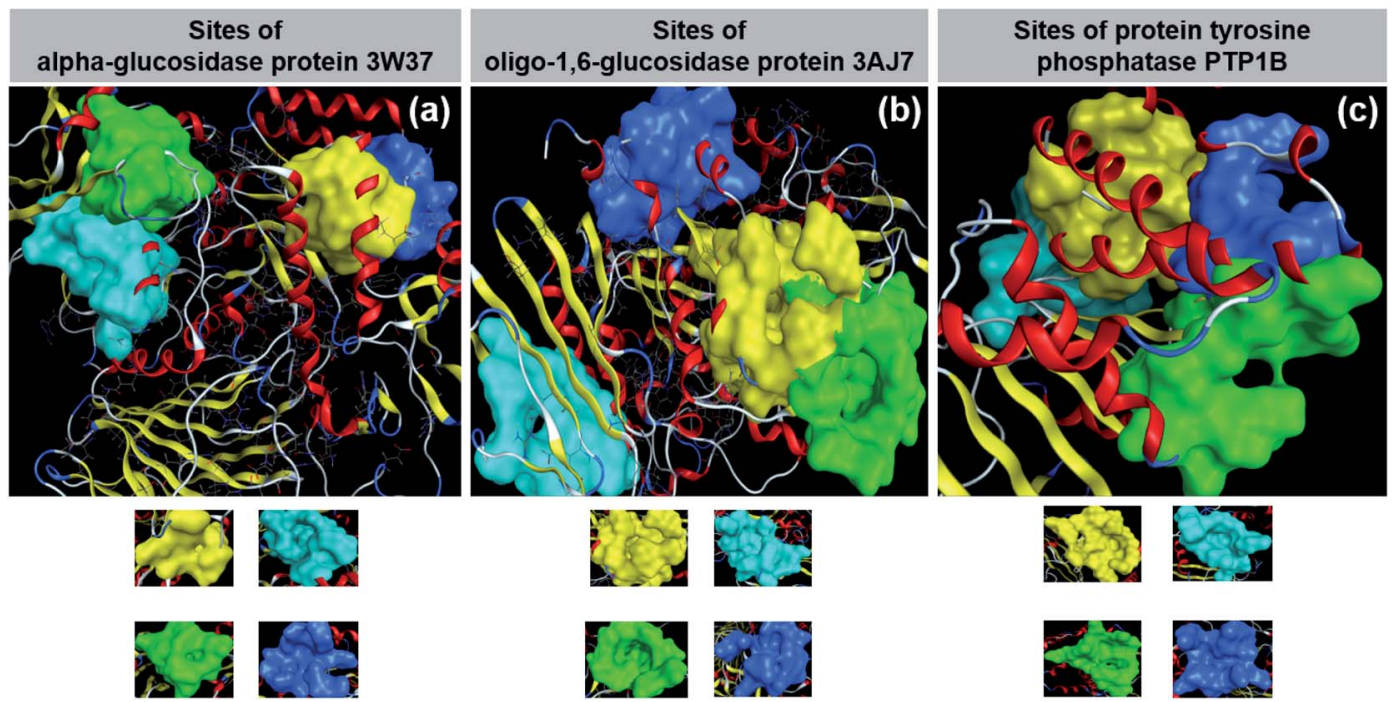

Fig. 4 Quaternary structures of proteins (a) 3W37, (b) 3AJ7 and (c) PTP1B with their approachable sites by investigated compounds (1, 2, 3a-3m, 4, 5, and 6a-6e): site 1 (yellow), site 2 (cyan), site 3 (green), and site 4 (blue).

its oxime ester derivatives (2, 3b, 3c, 3e, 3f, 3i, 3k, and $3 \mathbf{l})$ exhibited better the bio-activity than the standard compound acarbose towards $\alpha$-glucosidase, as shown by their $\mathrm{IC}_{50}$ values of $16.38-59.15 \mu \mathrm{M}$. The benzoate derivative (3c) is the most active compound in the oxime ester series with an $\mathrm{IC}_{50}$ of $16.38 \mu \mathrm{M}$. It is followed by the parachlorobenzoate $\left(3 \mathbf{f}, \mathrm{IC}_{50}\right.$ of $\left.23.98 \mu \mathrm{M}\right)$ and cinnamate $\left(3 \mathbf{b}, \mathrm{IC}_{50}\right.$ of $\left.33.12 \mu \mathrm{M}\right)$ derivatives. The substitution of monoiodine (3h) and diodine benzoate (3d) led to the loss of their activity. Compound $3 \mathbf{i}$ with meta-fluorobenzoate exhibited good activity with an $\mathrm{IC}_{50}$ value of $46.79 \mu \mathrm{M}$. However, the installation of para-fluorobenzoate $(\mathbf{3 g})$ resulted in no $\alpha$-glucosidase inhibition. The above-mentioned inhibitory effect suggests that the presence of the halogen substitutions in the aromatic rings increase the overall inhibition in the order of $\mathrm{Cl}$ $>\mathrm{F}>\mathrm{I}$. The heterocyclic oxime ester with a nicotinic ring (3e) showed good activity with an $\mathrm{IC}_{50}$ of $36.60 \mu \mathrm{M}$; in contrast, the pyrazine derivative was observed to be inactive. The acetate (3k) and pentanoate (3l) derivatives were found to exhibit good activity with the corresponding $\mathrm{IC}_{50}$ of 59.15 and $43.51 \mu \mathrm{M}$, respectively. The results suggest that the chain length of the alkoxy substitution may improve the $\alpha$-glucosidase inhibitory activity. The isobutyrate (3m) oxime ester was inactive in this test. This means that the bulkiness of the alkoxy oxime ester group seems to lower the activity overall.

Compound 4 with a lactone ring located at $\mathrm{C}-17$ was inactive to the $\alpha$-glucosidase inhibition; however, the reduction of the ketone to hydroxyl group at C-3 led to potential inhibitory activity. Compound $\mathbf{5}$ with a free hydroxyl group was registered as the most active compound ( $\mathrm{IC}_{50}$ of $2.73 \mu \mathrm{M}$ ) among the ester derivatives, as shown in Scheme 2. This evidence suggests that the hydroxyl group at C-3 plays an important role in $\alpha$-glucosidase inhibition. Sufficiently increasing the chain length and bulkiness of the alkanoate moieties may enhance the activity. This was evidenced by the increase in activity, which followed the order of: $6 \mathbf{a}\left(\mathrm{IC}_{50} 220.5 \mu \mathrm{M}\right)<\mathbf{6 b}\left(\mathrm{IC}_{50} 31.95 \mu \mathrm{M}\right)<\mathbf{6 c}\left(\mathrm{IC}_{50}\right.$
$4.62 \mu \mathrm{M}$ ) (Table 1). The cinnamate derivative showed significantly better activity than the salicylate one. This observation suggests that the electron density of the benzene rings may have a negative correlation with the inhibitory activity of the host molecules towards $\alpha$-glucosidase. Also, the dose-response curves of the most active inhibitors, i.e., 5, 6c, and $\mathbf{6 e}$, are provided in the ESI (Section 3; pages 203-205†).

In summary, it is obvious that the most significant inhibitors towards $\alpha$-glucosidase, which correspond to $\mathrm{IC}_{50}$ values lower than $10 \mu \mathrm{M}$, follow the order of $5>\mathbf{6 c}>\mathbf{6 e}$. In particular, 5 demonstrates pronounced efficacy with the corresponding value of $2.73 \pm 0.05 \mu \mathrm{M}$, accounting for the concentration required for $50 \%$ inhibition of the enzyme. This is followed by $3 \mathbf{c}>3 \mathbf{f}>6 \mathbf{b}>\mathbf{3 b}>\mathbf{3 e}>\mathbf{2}$, whose $\mathrm{IC}_{50}$ values are in the range of 10-40 $\mu \mathrm{M}$, and this considered highly effective. This group includes a mildly effective inhibitor, namely $\mathbf{3 e}$, which is expected to be very different according to the docking-based simulation. Also, the experiments found that $\mathbf{3 a}, \mathbf{3 d}, \mathbf{3 g}, \mathbf{3 h}$, 3m, and 4 exhibit no inhibition activity towards $\alpha$-glucosidase; nevertheless, the simulation gave the opposite prediction given 3d. The inconsistencies observed are readdressed and discussed in detail later in this report.

3.3.2. Computational simulation of diabetes-related protein inhibitability. The static interaction between the inhibitory agents (i.e., structure of the Dipterocarpus alatusrelated compounds) and the targeted proteins (viz., 3W37, 3AJ7, and PTP1B) was simulated using the molecular docking technique. The quaternary structure of the proteins and their approachable sites (by the ligands) are shown in Fig. 4. The docking results are summarised in Table 2, including the primary parameters, namely, the docking score (DS) energy, root-mean-square deviation (RMSD), number of hydrogen bonds, and number of van der Waals interactions. Detailed data is provided in the ESI (Sections 2.1-2.3; pages 194-202†). Overall, pre-screening of the two most important 


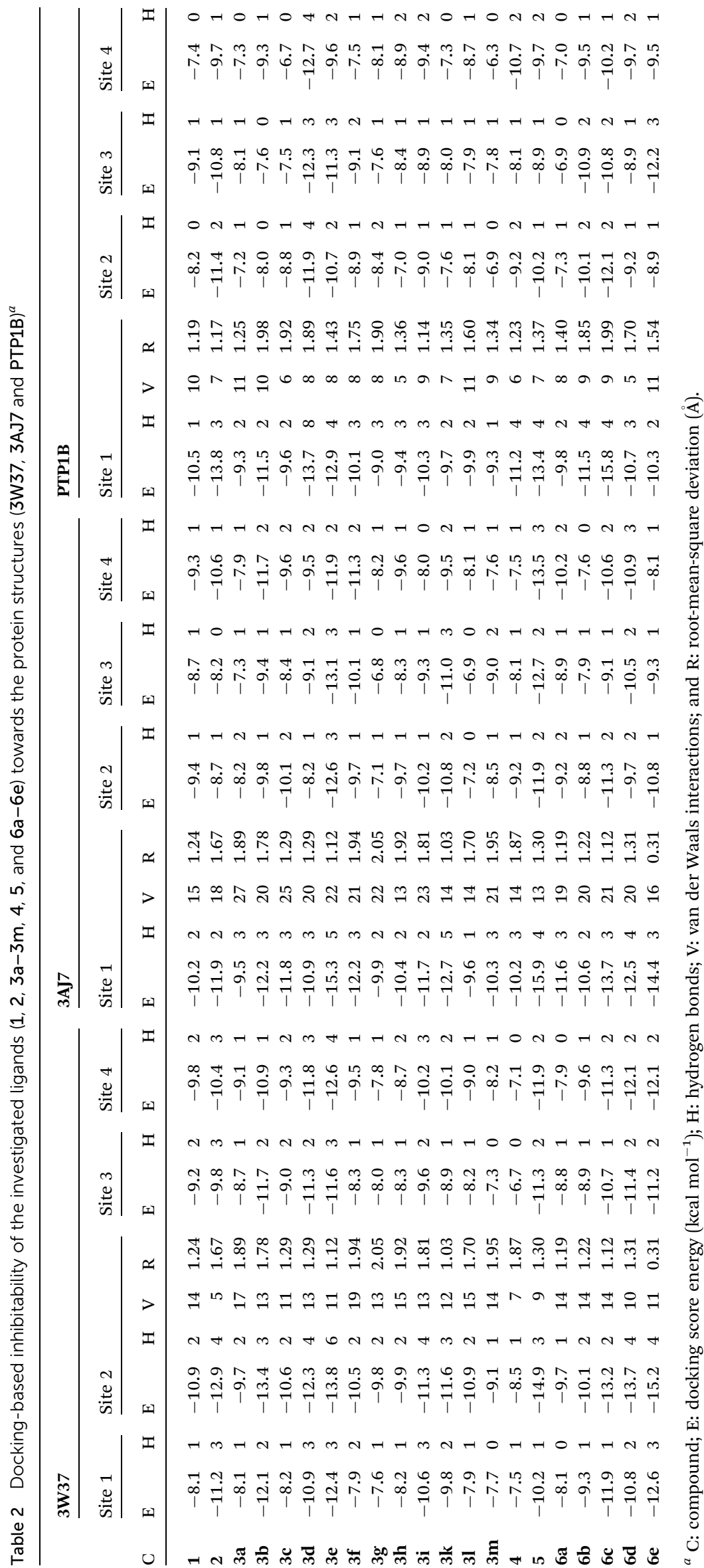


Table 3 QSARIS-based physicochemical properties of the studied ligands $(1,2,3 a-3 m, 4,5$, and $6 a-6 e)$ docked with the protein structures (3W37, 3AJ7 and PTP1B)

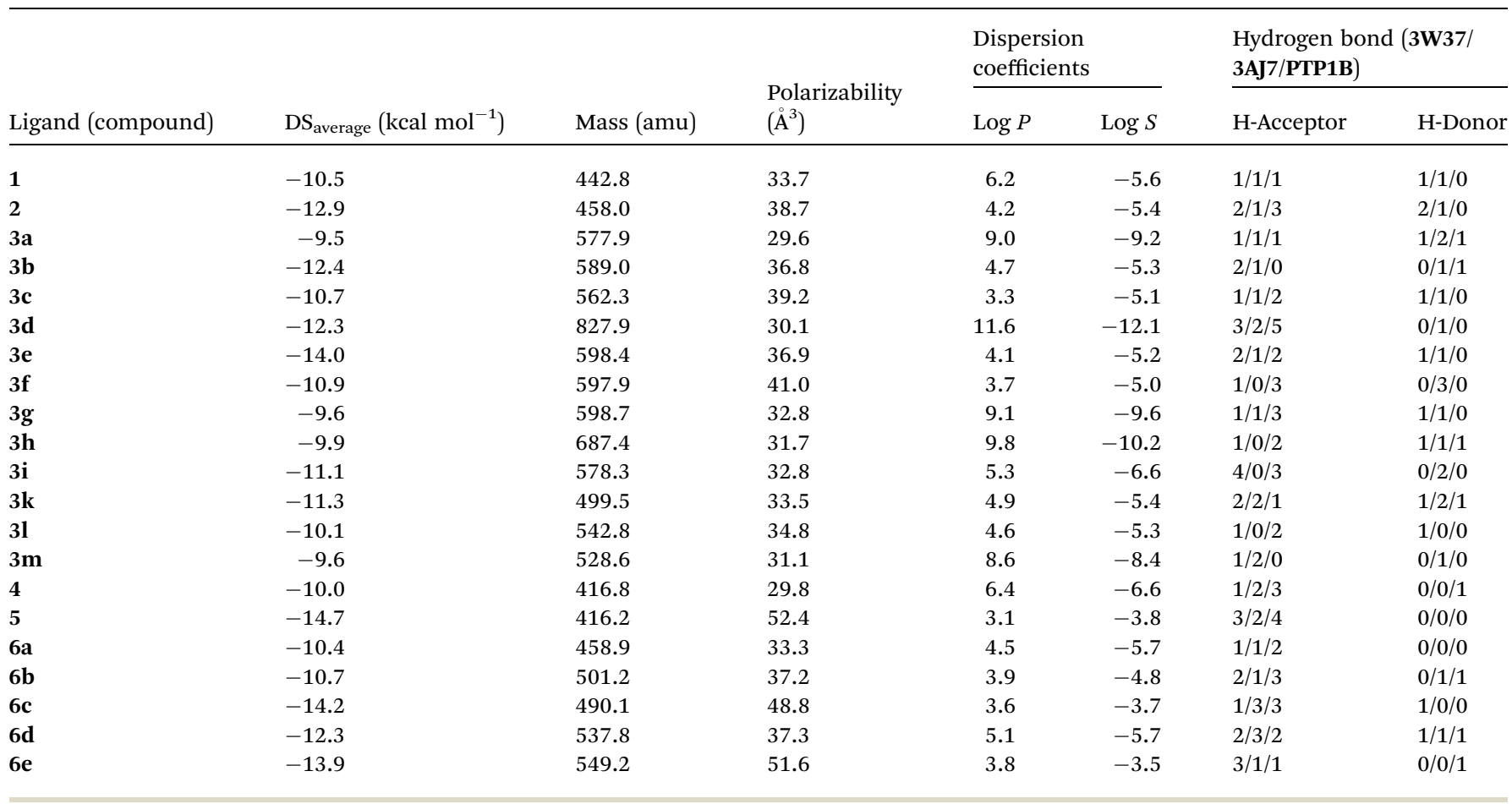

parameters, ${ }^{39-41,47}$ i.e., the DS values (representing static stability of an inhibitory system) and number of hydrogen bonds (representing the effectiveness of intermolecular binding, thus inducing conformational changes), indicated that 3W37, 3AJ7, and PTP1B are likely to be most susceptible at site 2 , site 1 , and site 1 , respectively. As an exception, $6 \mathbf{e}$ is predicted to exhibit the highest inhibition towards PTP1B if in its site 2. The corresponding figures selected for further in-depth analysis are presented in bold.

Regarding the investigated structure representing $\alpha$-glucosidase, viz., 3W37, the computational simulation is considered to be in good agreement with the experimental results collected from the bioassays. The docking-based computation predicted that the ligands with the highest inhibitability follow the order of $6 \mathrm{e}\left(\mathrm{DS}-15.2 \mathrm{kcal} \mathrm{mol}^{-1}\right)>5\left(\mathrm{DS}-14.9 \mathrm{kcal} \mathrm{mol}^{-1}\right)>3 \mathrm{e}(\mathrm{DS}$ $\left.-13.8 \mathrm{kcal} \mathrm{mol}{ }^{-1}\right)=6 \mathbf{d}\left(\mathrm{DS}-13.7 \mathrm{kcal} \mathrm{mol}^{-1}\right)>3 \mathbf{b}(\mathrm{DS}$ $\left.-13.4 \mathrm{kcal} \mathrm{mol}^{-1}\right)=6 \mathrm{c}\left(\mathrm{DS}-13.2 \mathrm{kcal} \mathrm{mol}^{-1}\right)$. The significance is highly justified by the values calculated using a similar computing configuration for three commercialised drugs, i.e., acarbose (DS $\quad-14.2 \mathrm{kcal} \quad \mathrm{mol}^{-1}$ ), voglibose (DS $-11.1 \mathrm{kcal} \mathrm{mol}^{-1}$ ), and miglitol (DS $-12.7 \mathrm{kcal} \mathrm{mol}^{-1}$ ), which were reported in our previous study. ${ }^{47}$ The corresponding RMSD values for the derivatives are all under $2 \AA$, indicating a biologically rigid conformation. ${ }^{48}$ Especially, that of $6 \mathrm{e}$ is $0.31 \AA$, meaning a significantly short average distance between its (backbone) atoms, and thus it can possibly be viewed as a quasicrystallised structure. All the compounds were determined to be effective $\alpha$-glucosidase inhibitors based on the assay experiments, except for 3e, which exhibited a moderate $\mathrm{IC}_{50}$ of $36.60 \pm$ $1.26 \mu \mathrm{M}$ in vitro although it was predicted to have the third lowest DS energy in silico. Furthermore, if the intermolecular interaction ability is considered, this ligand can create 6 strong hydrogen bonds, storing a total Gibbs free energy of $-10.3 \mathrm{kcal} \mathrm{mol}^{-1}$ (ESI: Section 2.1 and Table S1 $\dagger$ ), with the inpose amino acids of its targeted protein structure and should be highly conducive to the stability of the 3e-3W37 complex. Although observed in-discrete, this experiment-theory inconsistency is still notable, thus requiring further explanation. Alternatively, the compounds showing unnoticeable inhibition towards the enzyme in the in vitro investigation, viz., $\mathbf{3 a}, \mathbf{3 g}, \mathbf{3 h}$, $3 \mathbf{m}$, and $\mathbf{4}$, are also expected to form unstable ligand-protein complexes according to the static in silico study, given their significantly low DS values (over $-10 \mathrm{kcal} \mathrm{mol}^{-1}$ ). This excludes 3d, whose $\mathrm{IC}_{50}$ value is over $390 \mu \mathrm{M}$ (i.e., no activity) but its corresponding DS value is $-12.3 \mathrm{kcal} \mathrm{mol}^{-1}$ (i.e., considerable stability). In addition, 6d was experimentally demonstrated to be a weak $\alpha$-glucosidase inhibitor $\left(\mathrm{IC}_{50}\right.$ of $\left.228.09 \pm 4.62 \mu \mathrm{M}\right)$ but computationally predicted to exhibit good affinity towards the 3W37 structure (DS of $-13.7 \mathrm{kcal} \mathrm{mol}^{-1}$ ). Thus, these discrepancies also require appropriate explanations. Otherwise, the docking-based static models correspond well with the assaybased inhibition experiments.

However, due to the information lost from the pre-docking conditions, we performed a QSARIS-based analysis on the physicochemical properties of the studied ligands with reference to Lipinski's rule of five. The results are summarised in Table 3.

The first criterion regarding molecular mass ( $<500 \mathrm{amu})$ can be used to explain the docking-assay inconsistency. The corresponding values for ligands $\mathbf{3 d}$ and 3e are 827.9 and $598.4 \mathrm{amu}$, 


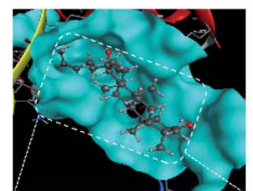

(2)

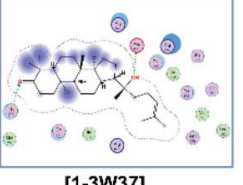

[1-3W37]
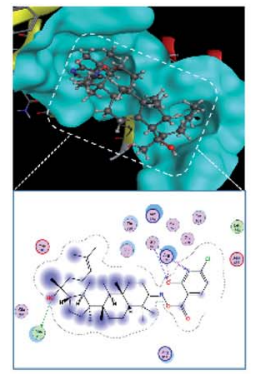

[3e-3W37]
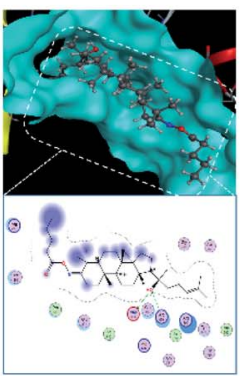

[31-3W37]
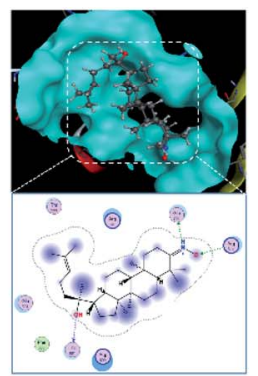

[2-3W37]
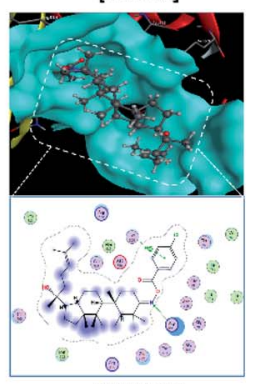

[3f-3W37]

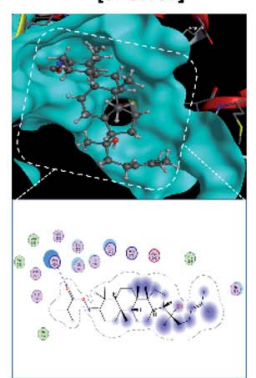

[3m-3W37]
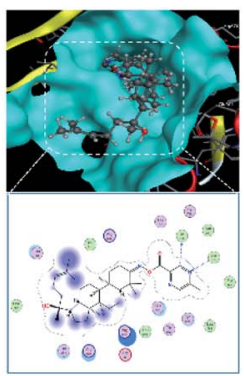

[3a-3W37]
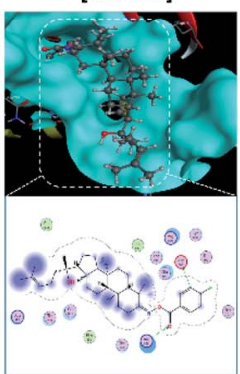

[3g-3W37]

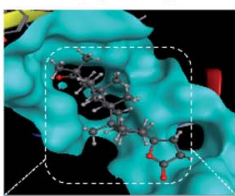$$
\text { (-) }
$$

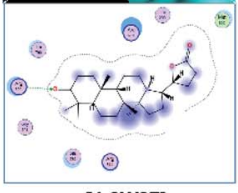

[4-3W37]
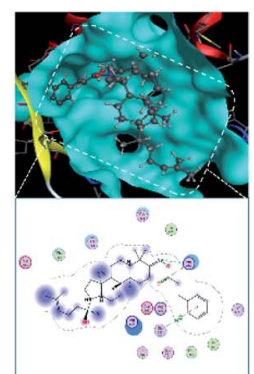

[3b-3W37]
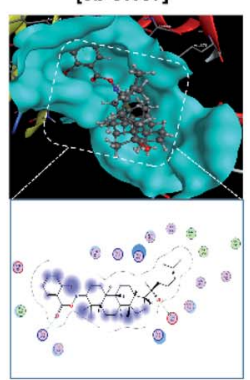

[3h-3W37]
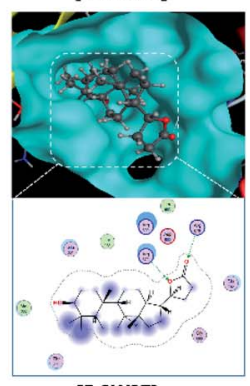

[5-3W37]

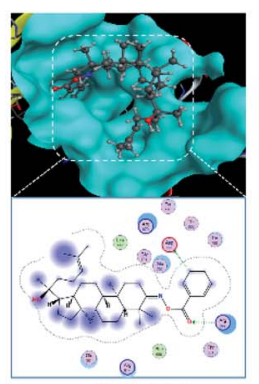

[3c-3W37]
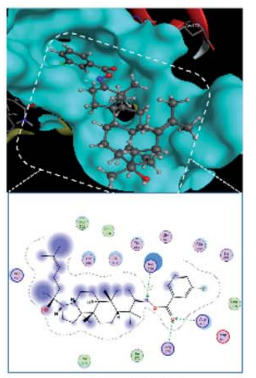

[3i-3W37]
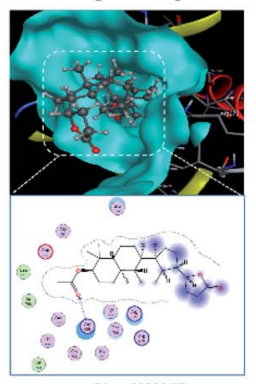

[6a-3W37]

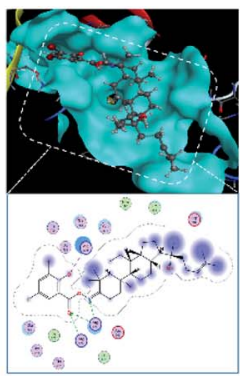

[3d-3W37]

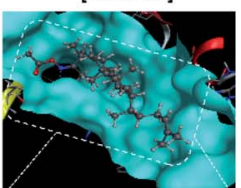

5.
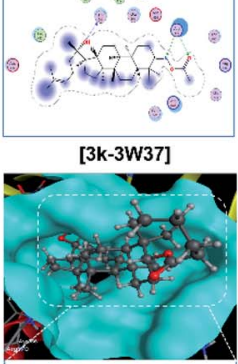

(2) $(9)$ 궁
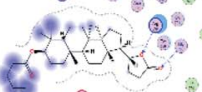

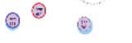

[6b-3W37]
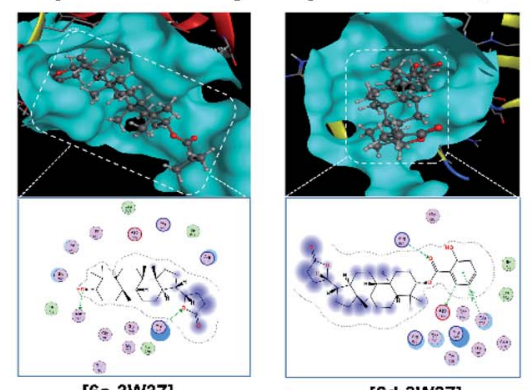

[6d-3W37]

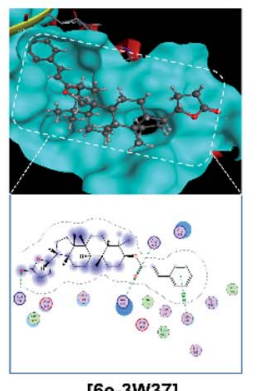

[6e-3W37]

Fig. 5 Visual presentation and in-pose interaction map of ligand-3W37 inhibitory complexes.

respectively. Their significant mass implies that even though they can exhibit inhibition towards the $\mathbf{3 W} 37$ structure when in the pose, their heavy mass is more likely to deter their transport from the medium of either the in vitro experiments or real physiological environment. A similar approach can explain the mild discrepancy observed in the case of $\mathbf{6 d}$ due to its slightly heavy mass of $537.8 \mathrm{amu}, c f$., Lipinski's criterion. In contrast, although having a lower-bound mass weight (mass of 461.8 amu), 4 exhibited no inhibition activity $\left(\mathrm{IC}_{50}>390 \mu \mathrm{M}\right)$ in the bioassay-based experiments, which is likely because of its low in-pose stability (DS of $-8.5 \mathrm{kcal} \mathrm{mol}^{-1}$ ). Otherwise, most of the assay-based inactive compounds, viz., 3a, 3d, 3g, 3h, and 3m, also possess a molecular mass over $500 \mathrm{amu}$, thus justifying this correlation. However, although $\mathbf{6 e}$ is predicted by the static model in the docking simulation to be the most promising inhibitor (DS of $-\mathbf{1 5 . 2} \mathrm{kcal} \mathrm{mol}^{-1}$ ) towards $\alpha$-glucosidase, the experimental observations reveal that it is only third-ranked $\left(\mathrm{IC}_{50}\right.$ of $\left.7.31 \pm 0.11 \mu \mathrm{M}\right)$, after $5\left(\mathrm{IC}_{50}\right.$ of $\left.2.73 \pm 0.05 \mu \mathrm{M}\right)$ and $6 \mathrm{c}\left(\mathrm{IC}_{50}\right.$ of $\left.4.62 \pm 0.12 \mu \mathrm{M}\right)$. In this case, the differences can be explained based on the slightly high mass of 6e, i.e. $549.2 \mathrm{amu}$, compromising its capability to form a highly stable 6e-3W37 inhibitory system, but not prohibitively. Besides molecular mass, according to Lipinski's criteria, a good membranepermeable molecule should satisfy the requirements of no more than 5 groups for hydrogen bonds; no more than 10 groups receiving hydrogen bonds; and a value of $\log P$ of less than $+5(\log P<5)$. Hence, it is obvious that the three most promising candidates, viz. 5, 6c, and $\mathbf{6 e}$, are ready for further 


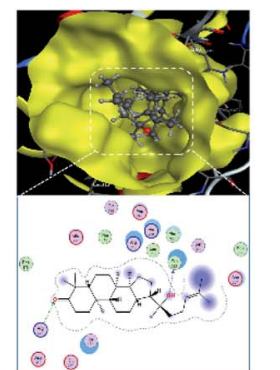

[1-3AJ7]

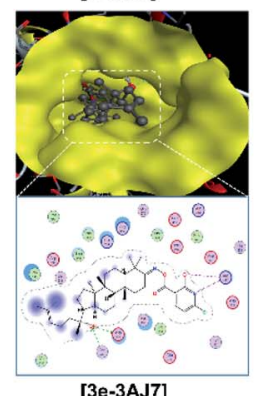

[3e-3AJ7]

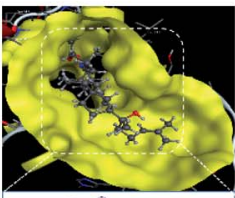

당

(3) $0^{\circ}$ क

$x+y<2$

$\therefore$ (3)

[31-3AJ7]

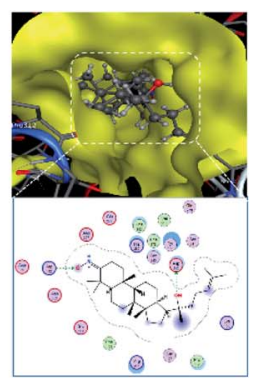

[2-3AJ7]

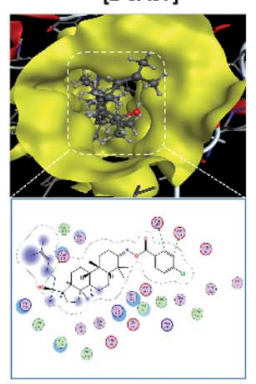

[3f-3AJ7]

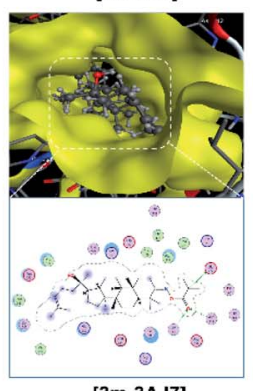

[3m-3AJ7]

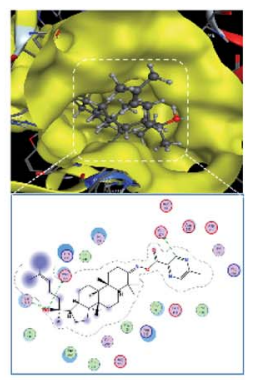

[3a-3AJ7]
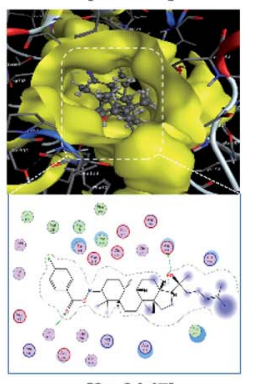

[3g-3AJ7]

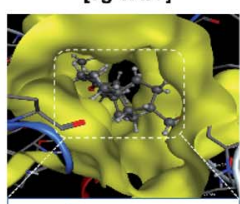

(ㄱ) 응

- $-12 x$

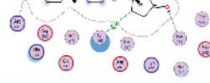

[4-3AJ7]

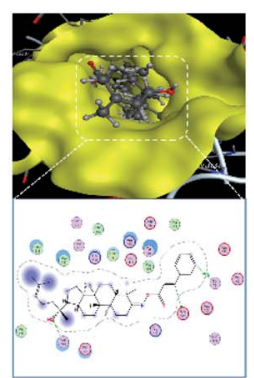

[3b-3AJ7]
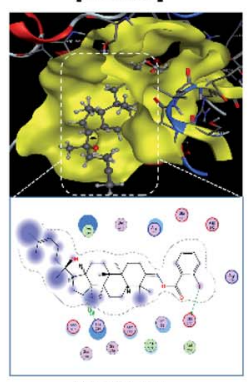

[3h-3AJ7]

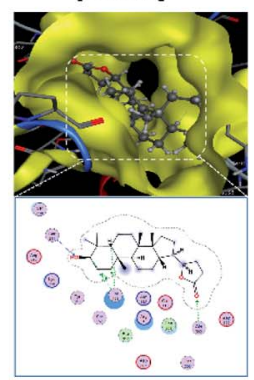

[5-3AJ7]

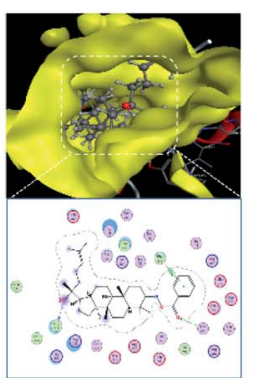

[3c-3AJ7]
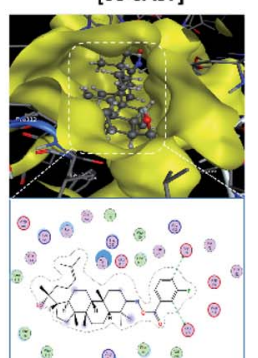

[3i-3AJ7]

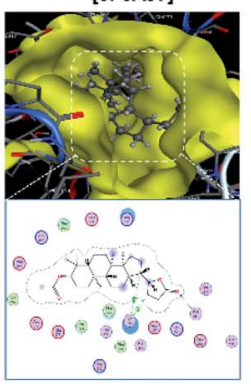

[6a-3AJ7]
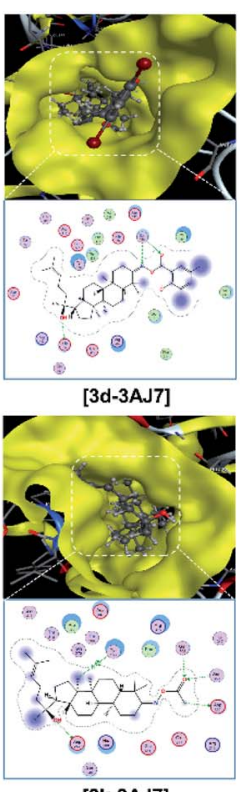

[3k-3AJ7]

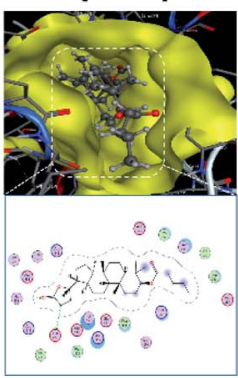

[6b-3AJ7]
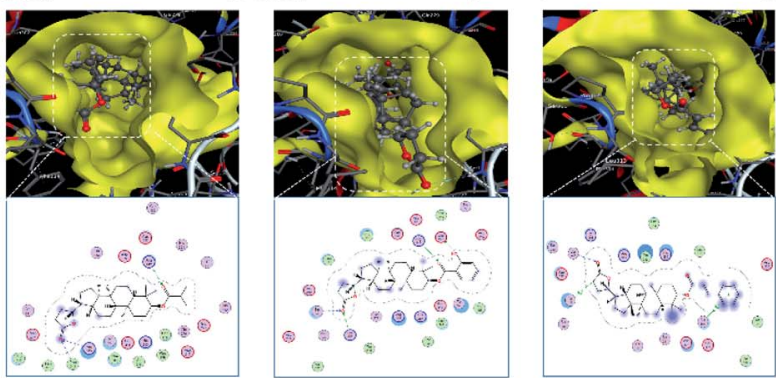

[6c-3AJ7]

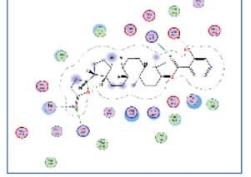

[6d-3AJ7]

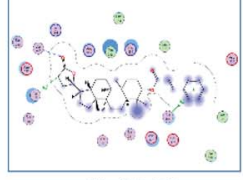

[6e-3AJ7]

Fig. 6 Visual presentation and in-pose interaction maps of the ligand-3AJ7 inhibitory complexes.

development in pharmaceutical applications, especially for oral administration. In addition, their polarisability constants are significant, i.e. $52.4 \AA^{3}$ for $\mathbf{5}, 48.8 \AA^{3}$ for $\mathbf{6 c}$, and $\AA^{3} 51.6$ for $\mathbf{6 e}$, likely inducing the formation of molecular dielectric moments. ${ }^{49}$ This property is thought to be highly conducive to both inhibitory effects towards a protein structure given that the polypeptide molecule is made of polarised amino acids and fluidity in hydrophilic environments of the body.

In summary, the molecular docking technique coupled with analysis of the QSARIS-based physicochemical properties can provide a reasonably accurate model to predict ligand-protein inhibitability in general, and dammarane- $\alpha$-glucosidase inhibition in particular. The latter determines the mobility of the ligands, whereas the former provides the stability of the ligand-protein complexes. This theoretical model suggests that the Dipterocarpus alatus-derived compounds are promising drug-like inhibitors towards $\alpha$-glucosidase, following the order of 5 (mass: $416.2 \mathrm{amu}$; polarisability: $52.4 \AA^{3}$; DS: $-14.9 \mathrm{kcal} \mathrm{mol}^{-1}$ ) > 6c (mass: 490.1 amu; polarisability: $48.8 \AA^{3}$; DS: $-13.7 \mathrm{kcal} \mathrm{mol}^{-1}$ ) > 6e (mass: $549.2 \mathrm{amu}$; polarisability: $51.6 \AA^{3}$; DS: $-15.2 \mathrm{kcal} \mathrm{mol}^{-1}$ ), which is in excellent agreement with the experiments. However, this model is still considered premature, thus requiring more solid-evidenced validations. Molecular dynamics simulation can monitor the simultaneous "behaviour" of all atoms in the systems, thus validating the mechanism of inhibition, and surface plasmon resonance can identify the correct ligand-protein complex structures.

Relatively, the inhibitability of the studied ligands towards the other diabetes-related protein structures, i.e., $3 \mathbf{A J} 7$ and 

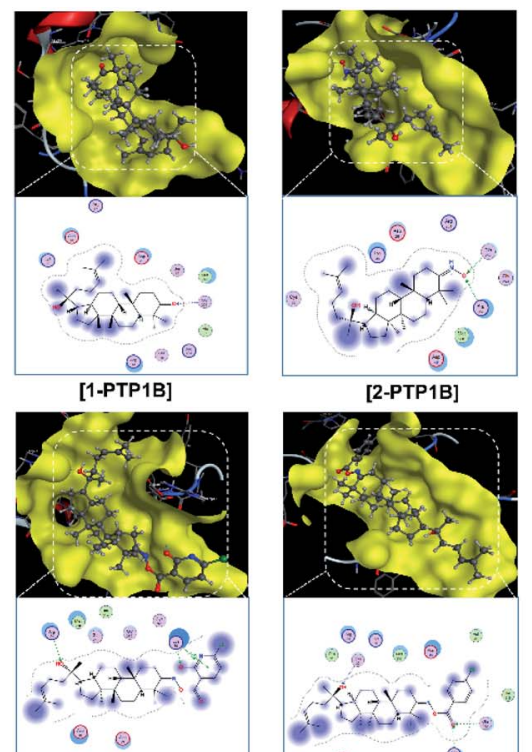

(2)

[3e-PTP1B]
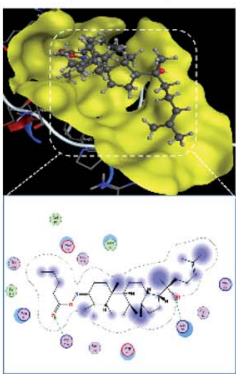

[3|-PTP1B7]
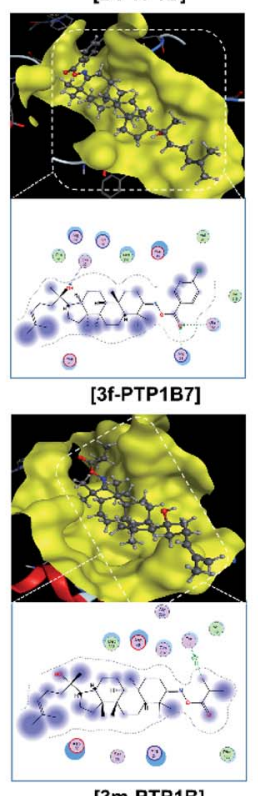
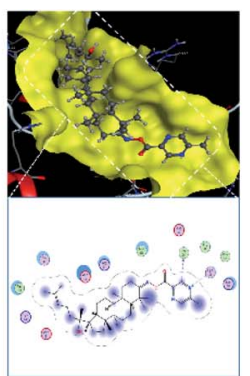

[3a-PTP1B]
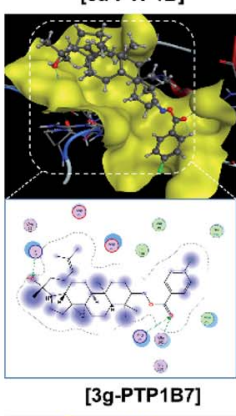

[3g-PTP1B

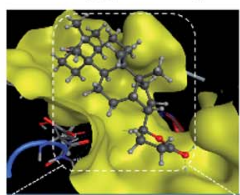

(1)

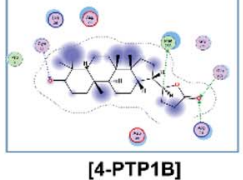

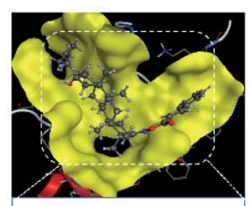

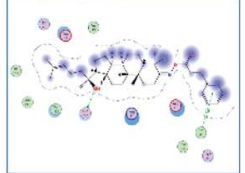

[3b-PTP1B]
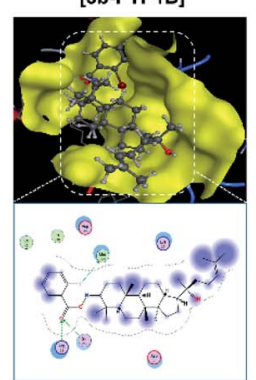

[3h-PTP1B]
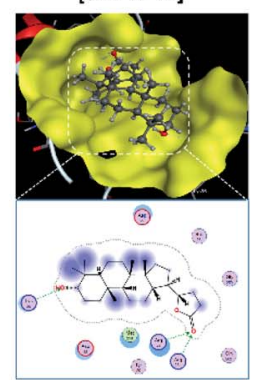

[5-PTP1B7]
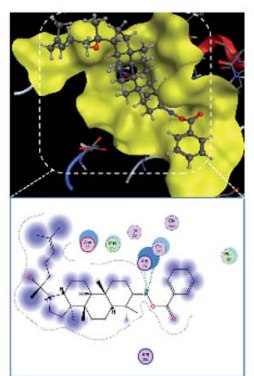

[3c-PTP1B]
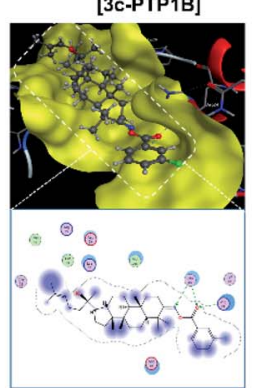

[3i-PTP1B]
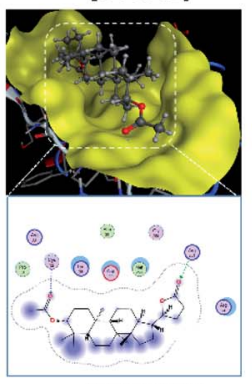

[6a-PTP1B7]
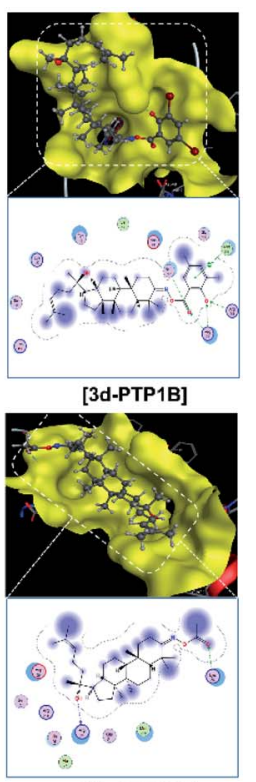

[3k-PTP1B7]
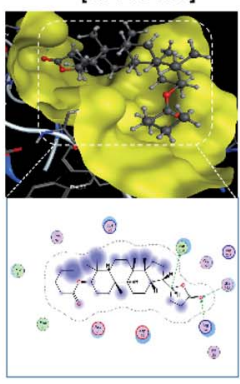

[6b-PTP1B]

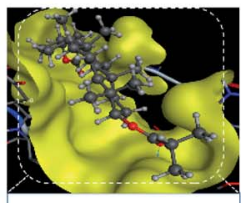

(c) $94 \times 4$ (2)

$2 x+7>2$
$0^{\circ}$

[6c-PTP1B]
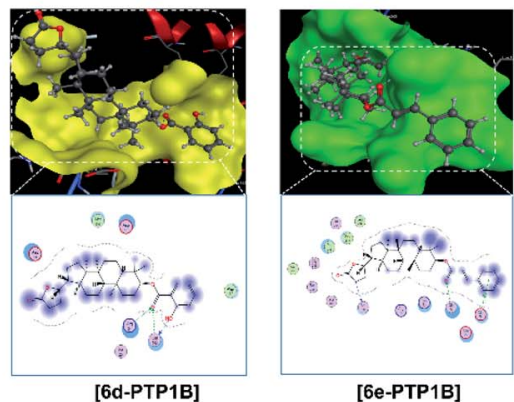

[6e-PTP1B]

Fig. 7 Visual presentation and in-pose interaction maps of the ligand-PTP1B7 inhibitory complexes.

PTP1B, was predicted without experimental evidence. Firstly, the former is still expected to be most vulnerable at its site 1 and with the strongest interactions derived by $\mathbf{5}$ (DS: $-15.9 \mathrm{kcal} \mathrm{mol}^{-1}$ ), 6c (DS: $-13.7 \mathrm{kcal} \mathrm{mol}^{-1}$ ), and 6e (DS: $-14.4 \mathrm{kcal} \mathrm{mol}^{-1}$ ). Although the 3e-3AJ7 inhibitory system has favourable stability, given its DS value of $-15.3 \mathrm{kcal} \mathrm{mol}^{-1}$, number of hydrogen bonds of 5 , and number of van der Waals interactions of 22, its formation its expected to be significantly hindered due to the heavy mass $(598.4 \mathrm{amu})$ and low polarisability $\left(36.9 \AA^{3}\right)$ of the ligand. Similarly, the formation of the 2PTP1B and 3d-PTP1B complexes, especially the latter, is almost unexpected despite the favourable stability of their intermolecular interaction. Besides, the dispersion coefficient $(\log P)$ of $\mathbf{3 d}$ is 11.6, which is much higher that the Lipinski's threshold, suggesting its insignificant dispersity. Meanwhile, 6e does not seem to be a promising inhibitor towards the PTP1B structure given its corresponding moderate DS values, regardless of the inhibited sites. Therefore, the preliminary analysis suggests that $\mathbf{5}$ and $\mathbf{6 c}$ are versatile inhibitors regarding all the diabetes-related protein structures, i.e., 3W37, 3AJ7, and PTP1B. The theoretical implications can be verified by appropriate experimental investigations, e.g., enzyme inhibition assays or clinical trials.

Finally, the 3D morphology and 2D interaction map of all the ligand-protein inhibitory complexes are visually presented in Fig. 5-7. In the design of specific protein inhibitors, the sizeand shape-compatibility between the ligands and the protein sites is also important. Although the Dipterocarpus alatusderived compounds are projected to be size-fitting (from the 3D 
observation) and shape-complementary (by 2D continuous proximity contours) with the in-pose topographical features of the diabetes-related protein structures, the sites are unlikely to be conducive to either the entry of significantly larger candidates or simultaneous inhibition. Therefore, a size- and medical-reference for the further development of pharmaceutical medicine are also given. In addition, the configurations of the in-pose interactions were projected in detail.

\section{Conclusions}

This study demonstrated the capability of Dipterocarpus alatusbased derivatives as effective inhibitors towards $\alpha$-glucosidase and proposed a new strategy to predict the ligand-protein inhibitability. Dipterocarpol (1) was isolated from Dipterocarpus alatus and 20 derivatives $(\mathbf{2}, \mathbf{3 a}-\mathbf{m}, \mathbf{4}, \mathbf{5}$, and $\mathbf{6 a - e})$ were obtained from semi-synthesis. Except for 2, the synthetic derivatives are new compounds. According to the experimental assay-based research, the most effective inhibitors towards $\alpha$ glucosidase follow the order of $5\left(\mathrm{IC}_{50}\right.$ of $\left.2.73 \pm 0.05 \mu \mathrm{M}\right)>\mathbf{6 c}$ $\left(\mathrm{IC}_{50}\right.$ of $\left.4.62 \pm 0.12 \mu \mathrm{M}\right)>6 \mathbf{e}\left(\mathrm{IC}_{50}\right.$ of $\left.7.31 \pm 0.11 \mu \mathrm{M}\right)$. Considering the computational docking-QSARIS analysis, the corresponding order is 5 (mass: $416.2 \mathrm{amu}$; polarisability: $52.4 \AA^{3}$; DS: $-14.9 \mathrm{kcal} \mathrm{mol}^{-1}$ ) > 6c (mass: $490.1 \mathrm{amu}$; polarisability: 48.8 $\AA^{3}$; DS: $-13.7 \mathrm{kcal} \mathrm{mol}^{-1}$ ) > 6e (mass: $549.2 \mathrm{amu}$; polarisability: $51.6 \AA^{3}$; DS $-15.2 \mathrm{kcal} \mathrm{mol}^{-1}$ ). These results indicate that the enzyme inhibition activity (observed from experiments) of a ligand highly correlates with its physicochemical properties (calculated by QSARIS and referenced to Lipinski's rule of five) and the ligand-protein static stability (simulated by molecular docking technique). The extended simulations predicted $\mathbf{5}$ and $\mathbf{6 c}$ to be versatile inhibitors regarding all the diabetes-related protein structures, viz. 3W37, 3AJ7, and PTP1B. These results can encourage further investigations to develop alternative antidiabetic drugs, and the prediction framework of ligand-protein inhibitability may attract more work for validation, e.g., surface plasmon resonance and molecular dynamics simulation.

\section{Conflicts of interest}

There are no conflicts to declare.

\section{Acknowledgements}

The research was financially supported by joint research project between Vietnam Academy of Science and Technology (VAST) and Russian (Project No. QTRU01.02/18-19) and Russian Foundation for Basic Research (project No. 18-53-54005). Nguyen Thi Ai Nhung also acknowledges the partial support of Hue University under the Core Research Program, Grant No. NCM.DHH.2020.04.

\section{References}

1 World Health Organization, Global report on diabetes, WHO Press, Geneva, Switzeland, 2016.
2 W. N. Washburn, Development of the renal glucose reabsorption inhibitors: A new mechanism for the pharmacotherapy of diabetes mellitus type 2, J. Med. Chem., 2009, 52, 1785-1794, DOI: 10.1021/jm8013019.

3 D. E. Moller, New drug targets for type 2 diabetes and the metabolic syndrome, Nature, 2001, 414, 821-827, DOI: 10.1038/414821a.

4 M. T. Ha, S. H. Seong, T. D. Nguyen, W. K. Cho, K. J. Ah, J. Y. Ma, M. H. Woo, J. S. Choi and B. S. Min, Chalcone derivatives from the root bark of Morus alba L. act as inhibitors of PTP1B and $\alpha$-glucosidase, Phytochemistry, 2018, 155, 114-125, DOI: 10.1016/j.phytochem.2018.08.001.

5 Z. Sun and C. A. Henson, Degradation of native starch granules by barley $\alpha$-glucosidases, Plant Physiol., 1990, 94, 320-327, DOI: 10.1104/pp.94.1.320.

6 H. Matsui, S. Chiba and T. Shimomura, Substrate specificity of an $\alpha$-glucosidase in sugar beet seed, Agric. Biol. Chem., 1978, 42, 1855-1860, DOI: 10.1080/00021369.1978.10863265.

7 M. Schaechter, Encyclopedia of microbiology, Academic Press, 2009.

8 K. Watanabe, Y. Hata, H. Kizaki, Y. Katsube and Y. Suzuki, The refined crystal structure of Bacillus cereus oligo-1,6glucosidase at $2.0 \AA$ resolution: Structural characterization of proline-substitution sites for protein thermostabilization, J. Mol. Biol., 1997, 269, 142-153, DOI: 10.1006/jmbi.1997.1018.

9 H. P. Hauri, A. Quaroni and K. J. Isselbacher, Biogenesis of intestinal plasma membrane: Posttranslational route and cleavage of sucrase-isomaltase, Proc. Natl. Acad. Sci. U. S. A., 1979, 76, 5183-5186, DOI: 10.1073/pnas.76.10.5183.

10 N. Krishnan, C. A. Bonham, I. A. Rus, O. K. Shrestha, C. M. Gauss, A. Haque, A. Tocilj, L. Joshua-Tor and N. K. Tonks, Harnessing insulin-and leptin-induced oxidation of PTP1B for therapeutic development, Nat. Commun., 2018, 9, 283, DOI: 10.1038/s41467-017-02252-2.

11 T. O. Johnson, J. Ermolieff and M. R. Jirousek, Protein tyrosine phosphatase 1B inhibitors for diabetes, Nat. Rev. Drug Discovery, 2002, 1, 696-709, DOI: 10.1038/nrd895.

12 R. A. Hill and J. D. Connolly, Triterpenoids, Nat. Prod. Rep., 2013, 30, 1028-1065, DOI: 10.1039/c3np70032a.

13 W. M. Bandaranayake, S. P. Gunasekera and S. Karunanayake, Terpenes of Dipterocarpus and Doona species, Phytochemistry, 1975, 14, 2043-2048, DOI: 10.1016/ 0031-9422(75)83122-0.

14 J. S. Mills and A. E. A. Werner, The chemistry of dammar resin, J. Chem. Soc., 1955, 3132-3140.

15 D. H. Kim, Chemical diversity of Panax ginseng, Panax quinquifolium, and Panax notoginseng, J. Ginseng Res., 2012, 36, 1-15, DOI: 10.5142/jgr.2012.36.1.1.

16 B. Dinda, S. Debnath, B. C. Mohanta and Y. Harigaya, Naturally occurring triterpenoid saponins, Chem. Biodiversity, 2010, 7, 2327-2580, DOI: 10.1002/ cbdv.200800070.

17 J. D. Connolly and R. A. Hill, Triterpenoids, Nat. Prod. Rep., 2008, 25, 794-830, DOI: 10.1039/b718038c.

18 L. Bai, J. Gao, F. Wei, J. Zhao, D. Wang and J. Wei, Therapeutic potential of ginsenosides as an adjuvant 
treatment for diabetes, Front. Pharmacol., 2018, 9, 1-14, DOI: 10.3389/fphar.2018.00423.

19 P. Zhou, W. Xie, S. He, Y. Sun, X. Meng, G. Sun and X. Sun, Ginsenoside $\mathrm{Rb} 1$ as an Anti-Diabetic Agent and Its Underlying Mechanism Analysis, Cells, 2019, 8, 204, DOI: 10.3390/cells8030204.

20 L. C. E. Lundqvist, D. Rattigan, E. Ehtesham, C. Demmou, C. G. Östenson and C. Sandström, Profiling and activity screening of Dammarane-type triterpen saponins from Gynostemma pentaphyllum with glucose-dependent insulin secretory activity, Sci. Rep., 2019, 9, 627, DOI: 10.1038/ s41598-018-37517-3.

21 D. Kim, S. Do, Y. Lee, M. Oh, J. Cha, T. Kim, F. Application and P. Data, Pharmaceutical composition for preventing and treating diabetes or glucose control abnormality comprising Ginsenosides, US pat., 7985848B2, 2011.

22 J. Cao, X. Zhang, F. Qu, Z. Guo and Y. Zhao, Dammarane triterpenoids for pharmaceutical use: A patent review (2005-2014), Expert Opin. Ther. Pat., 2015, 25, 805-817, DOI: $10.1517 / 13543776.2015 .1038239$.

23 E. F. Khusnutdinova, I. E. Smirnova, G. V. Giniyatullina, N. I. Medvedeva, E. Y. Yamansarov, D. V. Kazakov, O. B. Kazakova, P. T. Linh, D. Q. Viet and D. T. Huong, Inhibition of alpha-glucosidase by synthetic derivatives of lupane, oleanane, ursane and dammarane triterpenoids, Nat. Prod. Commun., 2016, 11, 33-35, DOI: 10.1177/ $1934578 \times 1601100112$.

24 L. D. Shao, Y. Bao, Y. Shen, J. Su, Y. Leng and Q. S. Zhao, Synthesis of selective 11ß-HSD1 inhibitors based on dammarane scaffold, Eur. J. Med. Chem., 2017, 135, 324338, DOI: 10.1016/j.ejmech.2017.04.059.

25 J. Q. Xu, Q. Shen, J. Li and L. H. Hu, Dammaranes from Gynostemma pentaphyllum and synthesis of their derivatives as inhibitors of protein tyrosine phosphatase 1B, Bioorg. Med. Chem., 2010, 18, 3934-3939, DOI: 10.1016/ j.bmc.2010.04.073.

26 P.-H. Ho, An illustrated flora of Vietnam 1, Youth Publishing House, Hanoi, 1999.

27 I. E. Smirnova, O. B. Kazakova, D. T. T. Huong, E. M. Minnibaeva, A. N. Lobov and K. Y. Suponitsky, Onepot synthesis of hollongdione from dipterocarpol, Nat. Prod. Commun., 2014, 9, 1417-1420, DOI: 10.1177/ $1934578 x 1400901005$.

28 I. E. Smirnova and T. T. P. Thao, Evaluation on antimicrobial potential of dipterocarpol and dammarenolic acid derivatives from Dipterocarpus alatus, Vietnam J. Chem., 2020, 58, 410-416, DOI: 10.1002/vjch.202000038.

29 O. B. Kazakova, I. E. Smirnova, N. I. Medvedeva, T. V. Lopatina, I. V. Chudov, A. R. Sharipov, A. S. Ziganshin and T. T. P. Thao, Hepatoprotective Activity of Betulin and Dipterocarpol Derivatives, Russ. J. Bioorg. Chem., 2019, 45, 558-565, DOI: 10.1134/S1068162019050030.

30 D. T. T. Huong, T. T. T. Thuy, T. T. Hien, N. T. Tra, N. Q. Tien, I. E. Smirnova, O. B. Kazakova, E. M. Minnibaeva and G. A. Tolstikov, Synthesis and Cytotoxicity of Derivatives of Dipterocarpol, a Metabolite of Dipterocarpus alatus, Chem.
Nat. Compd., 2013, 49, 58-65, DOI: 10.1007/s10600-0130505-4.

31 B. Bednarczyk-Cwynar and L. Zaprutko, Recent advances in synthesis and biological activity of triterpenic acylated oximes, Phytochem. Rev., 2015, 14, 203-231, DOI: 10.1007/ s11101-014-9353-5.

32 D. Li, J. Cao, X. Bi, X. Xia, W. Li and Y. Zhao, New dammarane-type triterpenoids from the leaves of Panax notoginseng and their protein tyrosine phosphatase $1 \mathrm{~B}$ inhibitory activity, J. Ginseng Res., 2014, 38, 28-33, DOI: 10.1016/j.jgr.2013.11.013.

33 M. A. F. Nasution, E. P. Toepak, A. H. Alkaff and U. S. F. Tambunan, Flexible docking-based molecular dynamics simulation of natural product compounds and Ebola virus Nucleocapsid (EBOV NP): A computational approach to discover new drug for combating Ebola, $B M C$ Bioinf., 2018, 19, 419, DOI: 10.1186/s12859-018-2387-8.

34 L. H. S. Santos, R. S. Ferreira and E. R. Caffarena, Integrating molecular docking and molecular dynamics simulations, Methods Mol. Biol., 2019, 2053, 13-34, DOI: 10.1007/978-14939-9752-7_2.

35 R. Priya, R. Sumitha, Cg. P. Doss, C. Rajasekaran, S. Babu, R. Seenivasan and R. Siva, Molecular docking and molecular dynamics to identify a novel human immunodeficiency virus inhibitor from alkaloids of Toddalia asiatica, Pharmacogn. Mag., 2015, 11, 414, DOI: 10.4103/0973-1296.168947.

36 D. R. Cameron, Computer Software Reviews, J. Am. Chem. Soc., 2001, 123, 8644-8645, DOI: 10.1021/ja015228g.

37 Y. M. Kim, M. H. Wang and H. I. Rhee, A novel $\alpha$-glucosidase inhibitor from pine bark, Carbohydr. Res., 2004, 339, 715717, DOI: 10.1016/j.carres.2003.11.005.

38 A. Duraiswamy, D. Shanmugasundaram, C. S. Sasikumar, S. M. Cherian and K. M. Cherian, Development of an antidiabetic formulation (ADJ6) and its inhibitory activity against $\alpha$-amylase and $\alpha$-glucosidase, J. Tradit. Complement. Med., 2016, 6, 204-208, DOI: 10.1016/j.jtcme.2014.12.006.

39 T. Du Ngo, T. D. Tran, M. T. Le and K. M. Thai, Computational predictive models for P-glycoprotein inhibition of in-house chalcone derivatives and drug-bank compounds, Mol. Diversity, 2016, 20, 945-961, DOI: 10.1007/s11030-016-9688-5.

40 T. M. Chandra Babu, S. S. Rajesh, B. V. Bhaskar, S. Devi, A. Rammohan, T. Sivaraman and W. Rajendra, Molecular docking, molecular dynamics simulation, biological evaluation and 2D QSAR analysis of flavonoids from Syzygium alternifolium as potent anti-Helicobacter pylori agents, RSC Adv., 2017, 7, 18277-18292, DOI: 10.1039/ c6ra27872h.

41 K. M. Thai, D. P. Le, N. V. K. Tran, T. T. H. Nguyen, T. D. Tran and M. T. Le, Computational assay of Zanamivir binding affinity with original and mutant influenza neuraminidase 9 using molecular docking, J. Theor. Biol., 2015, 385, 3139, DOI: 10.1016/j.jtbi.2015.08.019.

42 O. Tarasova, V. Poroikov and A. Veselovsky, Molecular Docking Studies of HIV-1 Resistance to Reverse 
Transcriptase Inhibitors: Mini-Review, Molecules, 2018, 23, 11-13, DOI: 10.3390/molecules23051233.

$43 \mathrm{~J}$. Gasteiger and M. Marsili, Iterative partial equalization of orbital electronegativity-a rapid access to atomic charges, Tetrahedron, 1980, 36, 3219-3228, DOI: 10.1016/00404020(80)80168-2.

44 C. A. Lipinski, F. Lombardo, B. W. Dominy and P. J. Feeney, Experimental and computational approaches to estimate solubility and permeability in drug discovery and development settings, Adv. Drug Delivery Rev., 1997, 23, 325, DOI: 10.1016/S0169-409X(96)00423-1.

45 M. J. Ahsan, J. G. Samy, H. Khalilullah, M. S. Nomani, P. Saraswat, R. Gaur and A. Singh, Molecular properties prediction and synthesis of novel 1,3,4-oxadiazole analogues as potent antimicrobial and antitubercular agents, Bioorg. Med. Chem. Lett., 2011, 21, 7246-7250, DOI: 10.1016/j.bmcl.2011.10.057.

46 J. Mazumdera, R. Chakraborty, S. Sena, S. Vadrab, B. Dec and T. K. Ravi, Synthesis and biological evaluation of some novel quinoxalinyl triazole derivatives, Der Pharma Chem., 2009, 1, 188-198.

47 T. Thi, P. Thao, T. Q. Bui, T. Quy, C. Bao, T. Van, T. T. Phuong Thao, T. Q. Bui, P. T. Quy, N. C. Bao, T. Van Loc, T. Van Chien, N. L. Chi, N. Van Tuan, T. Van Sung and N. T. Ai Nhung, Isolation, semi-synthesis, docking-based prediction, and bioassay-based activity of Dolichandrone spathaceairidoids: new catalpol derivatives as glucosidase inhibitors, RSC Adv., 2021, 11, 11959-11975, DOI: 10.1039/ d1ra00441g.

48 Y. Ding, Y. Fang, J. Moreno, J. Ramanujam, M. Jarrell and M. Brylinski, Assessing the similarity of ligand binding conformations with the Contact Mode Score, Comput. Biol. Chem., 2016, 64, 403-413, DOI: 10.1016/ j.compbiolchem.2016.08.007.

49 R. Feynman, The Feynman lectures on physics - Volume II, Millenium, Basic Books, New York, 2010. 